\title{
PONTIC SIGILLATA DISCOVERED IN THE GETO-DACIAN SETTLEMENT OF POIANA, NICOREŞTI COMMUNE, GALAȚI COUNTY
}

Mariana-Cristina Popescu

\section{Sigillatele pontice descoperite în aşezarea geto-dacică de la Poiana, comuna Nicoreşti, jud. Galați}

Vesela elenistică şi romană descoperită în aşezarea geto-dacică de la Poiana (com. Nicoreşti, jud. Galați, România) şi în mare parte aflată în colecția Muzeului Orăşenesc din Tecuci, jud. Galaţi, a fost prezentată de-a lungul timpului în rapoartele de săpătură, în articole şi în monografia dedicată sitului şi, de asemenea, în studii dedicate importurilor elenistice şi romane descoperite pe teritoriul Daciei preromane sau într-un studiu special dedicat ceramicii de import din aşezarea amintită. O reevaluare a ceramicii descoperite în situl de la Poiana a permis identificarea mai multor clase de ceramică elenistică şi romană, dintre care sigillatele pontice fac subiectul prezentului articol. Din lotul de vase elenistice şi romane pe care le-am avut la dispoziție pentru studiu am identificat 184 de sigillate pontice, piese întregi şi întregibile, ori fragmente de vase cu decor specific acestei clase de sigillate care, din punct de vedere funcțional, se pot grupa în două categorii: vase pentru servit şi vase pentru băut. În ceea ce priveşte formele de vase, am identificat patru forme Hayes (Hayes I, Hayes V, Hayes VIII si Hayes X) şi trei forme care, pe baza analogiilor nord-pontice şi a pastei similare cu a formelor Hayes, pot fi atribuite aceleiaşi clase ceramice.

Key words: Roman pottery, Pontic sigillata, Geto-Dacian settlements, North Pontic sites, Romania.

Pontic sigillata, a pottery class manufactured during the $1^{\text {st }}$ and the end of the $3^{\text {rd }} \mathrm{c}$. AD by the workshops in the basin of the Black Sea, is well documented in sites north of Pontus and also signalled in sites from Romania and Bulgaria ${ }^{1}$.

The centre or centres manufacturing such pottery remain unknown for the time being. Although they are regular in the aforementioned sites during the period between the end of the $1^{\text {st }} \mathrm{c}$. BC $-2^{\text {nd }} \mathrm{c}$. $\mathrm{AD}$, currently, there is no sufficient data that would establish their origin from one specific site or, at least, specific area. Consistent information related to the sites in northern Turkey (Pontus and ancient Bithynia) ${ }^{2}$ are missing; in Histria's case, one may not clearly ascertain the existence of the own production of luxury pottery during the Roman period ${ }^{3}$; in the case of the sites in the north of the Black Sea, current state of research is defined as an intense period of material collection and issue of preliminary studies and it is estimated that: "in a few years time, it will be possible to complete a detailed classification of Pontic sigillata from the North Pontic region"4.

First shapes of Pontic sigillata were classified by T. N. Knipovich on the occasion of processing the red-slipped pottery uncovered at Olbia, Tiritake and Mirmekion, which were

\footnotetext{
${ }^{1}$ Atlante II, 92-93; Zhuravlev 2002, 254-261.

${ }^{2}$ Atlante II, 92.

${ }^{3}$ Suceveanu 2002, 179.

${ }^{4}$ Zhuravlev 2002, 255.
} 
attributed to the large centres known at that time: Arezzo, Samos and Pergamum ${ }^{5}$. Kropotkin also publishes a few exemplars belonging to this class under the name of red-slipped Bosporan pottery"6. J. W. Hayes drafts the first basic classification of Pontic sigillata based on Knipovich and Kropotkin publications ${ }^{7}$.

D. Zhuravlev proposes three typological series for this sigillata category differentiated upon shapes and chronology as follows: Pontic sigillata A, massively diffused during the second half of the $1^{\text {st }} \mathrm{c}$. to the first half of the $2^{\text {nd }} \mathrm{c}$. AD; Pontic sigillata $B$, manufactured from mid $2^{\text {nd }}$ c. and Pontic sigillata $C$, registering a period of maximum distribution during the $2^{\text {nd }}-$ $3^{\text {rd }}$ c. $\mathrm{AD}^{8}$. The cited author does not provide many details regarding the shapes included in each typological series, limiting to only technical - fabric, slip, decoration, firing - and chronological information and publishes only a few plates comprising most representative shapes of the first two typological series, while the majority of Hayes shapes are included in the Pontic sigillata A series?.

To Pontic sigillata are characteristically tableware: drinking ware (cups, beakers, kantharoi), pouring ware (lagynoi, jars, jugs) and service tableware (plates, bowls). Additionally, the decoration made by barbotine technique present on lagynoi and drinking ware seems to be specific to Pontic sigillata ${ }^{10}$.

Pontic sigillata are rarely found outside the basin of the Black Sea and very few items were signalled in Athens, Corinth or northern Africa ${ }^{11}$. A small number of additional exemplars were discovered in Ostia, yet they are considered exceptions not indicative for any trade activity, but which rather mirror the relations range this port held ${ }^{12}$.

The items included in the catalogue were discovered following archaeological systematic excavations carried out in the Geto- Dacian site of Poiana (fig. 1, fig 2) and come almost exclusively from the collections housed with the City Museum of Tecuci, Galaţi district $^{13}$. A Hayes X kantharos (40) in the collection housed with the former Museum of Antiquities of Bucharest, published by I. Glodariu, is an exception and we described it based on bibliographical information ${ }^{14}$.

Part of the items were published within excavation reports or special articles dedicated to the settlement ${ }^{15}$, yet they are difficult, if not impossible to identify. The items uncovered until 1969 were analysed and interpreted by I. Glodariu ${ }^{16}$, while a selection of pottery coming from Poiana was published in a special article dedicated to this material category ${ }^{17}$ and additionally, in the site monograph ${ }^{18}$.

\footnotetext{
${ }^{5}$ Atlante II, 92.

${ }^{6}$ Atlante II, 92

${ }^{7}$ Atlante II, 92-96, tav. XXIII-XXIV.

${ }^{8}$ Zhuravlev 2002, 256-261.

${ }^{9}$ Zhuravlev 2002, 256-261, fig. 12-16.

${ }^{10}$ Atlante II, 92-93.

${ }^{11}$ Atlante II, 93; Zhuravlev 2002, 254.

${ }^{12}$ Atlante II, 93.

${ }^{13}$ I would like to thank Mrs. Silvia Teodor for amiable approval of my observing and processing of the items coming from the site at Poiana discovered following the archaeological excavations she carried out in this site or coming from previous digs; I would also like to thank the management of the Museum of Tecuci and its custodians for their help during my research.

${ }^{14}$ Glodariu 1974, 215, cat. 34/35, pl. XXIV, c 34/35.

${ }^{15}$ Vulpe, Vulpe 1924, 166-223; Vulpe 1955, 239-271.

${ }^{16}$ Glodariu 1973, the catalogue of import pottery, p. 214-216.

${ }_{17}$ Teodor 1994, 73-123.

${ }^{18}$ Vulpe, Teodor 2003, 321-334, fig. 235-241.
} 


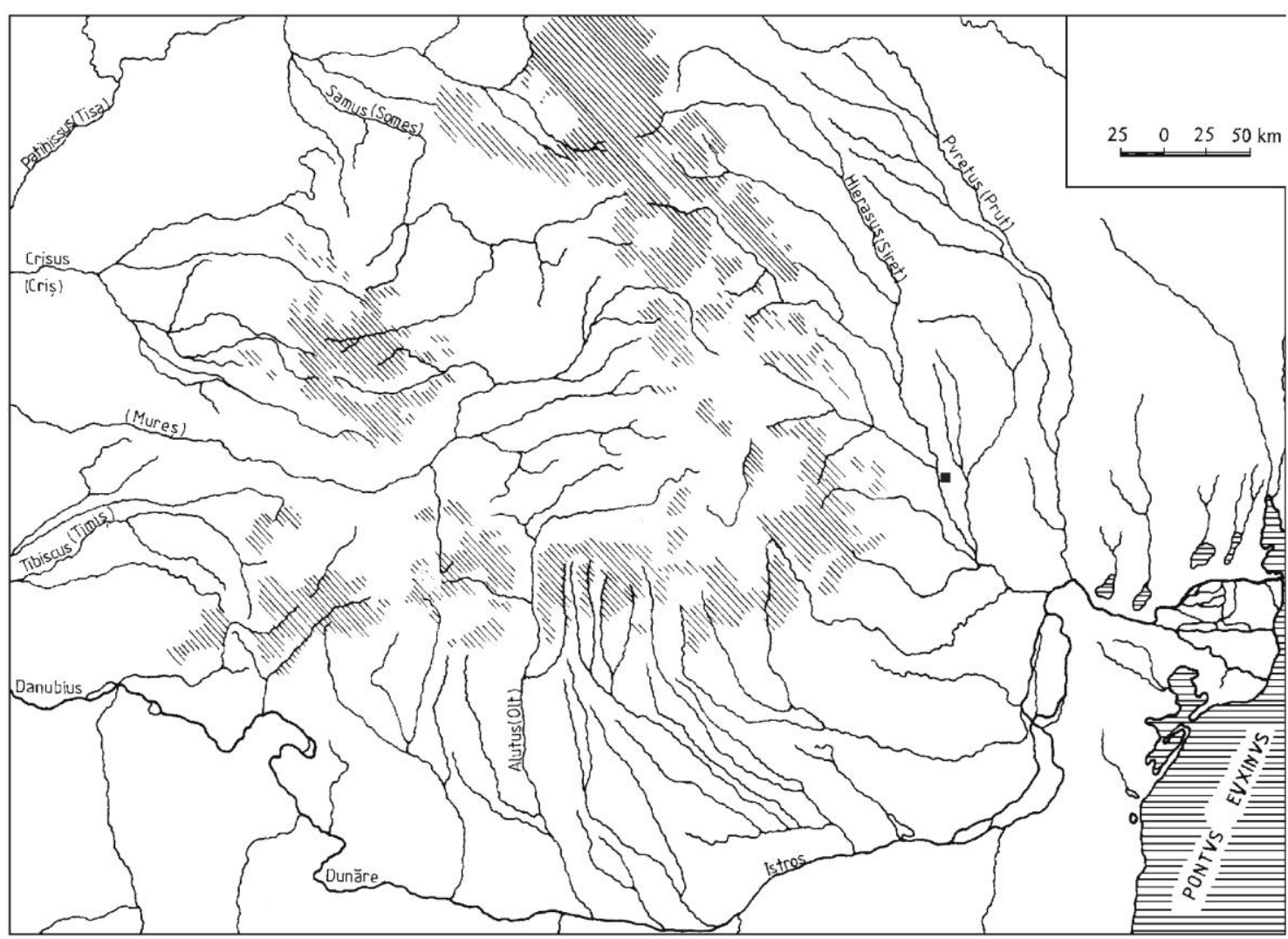

Fig. 1. Romanias map with Geto-dacian settlement of Poiana

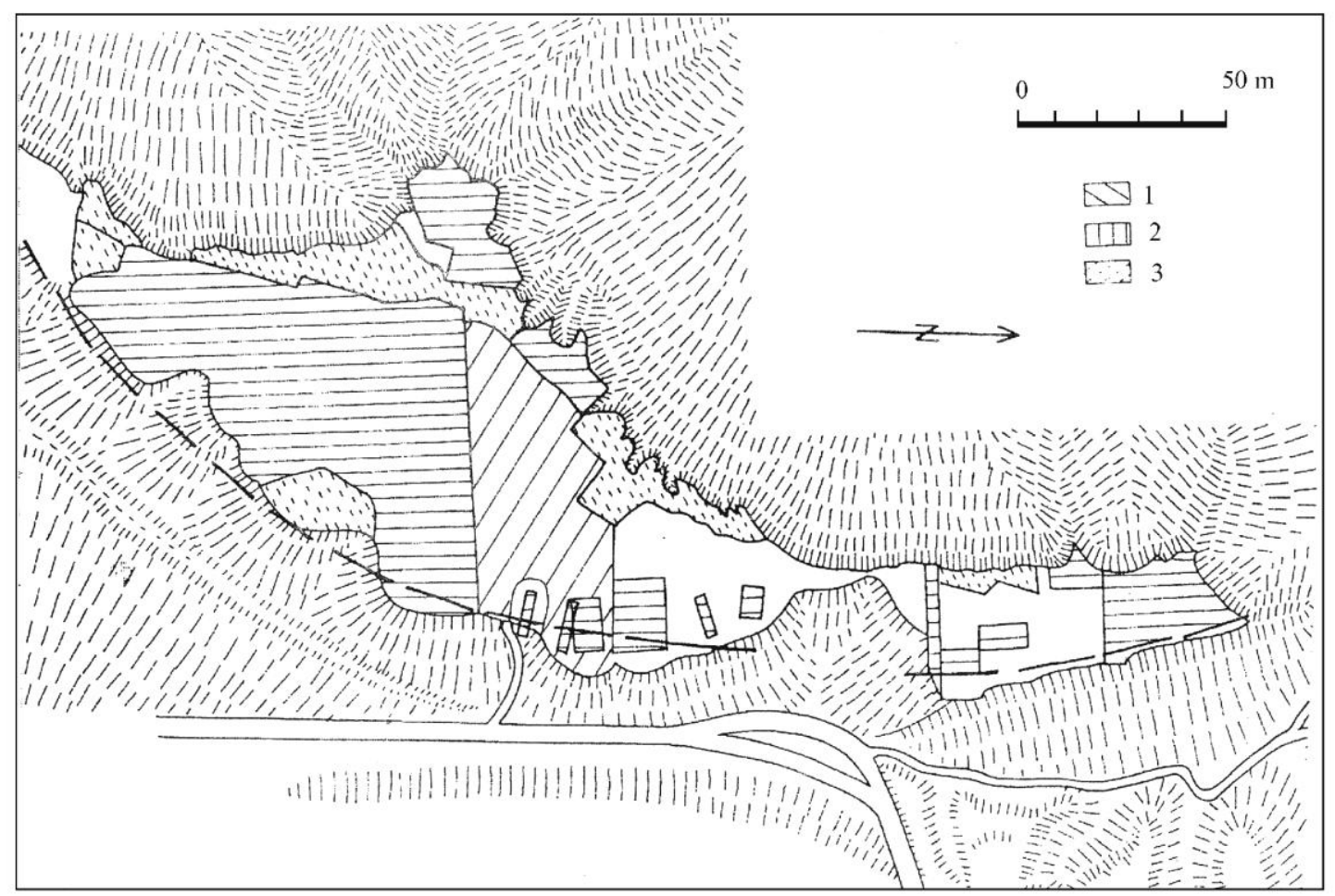

Fig. 2. Topographic plan of Poiana settlement: 1. excavations from 1985-1990; 2. excavations from 1926 to $1951 ; 3$. sondages (after Vulpe, Teodor 2003, fig. 1) 
Unfortunately many of the information regarding the context wherein the items were discovered is no longer preserved ${ }^{19}$, therefore we may not possess detailed observations regarding the circulation of Pontic sigillata in the site at Poiana. Moreover, in numerous cases, the inventory number and mark of the discovery context written onto the item do not correspond with the information entered into the inventory register. Even so, I preferred to record in the catalogue all data marked on the pieces, so a future identification would be made straightforwardly.

From the lot of Hellenistic and Roman items available to me, I succeeded in identifying a number of 184 Pontic sigillata - complete and restorable vessels or vessel fragments displaying decoration specific to Pontic sigillata. The material is fragmentary, yet in many cases the profile is complete or almost complete and the shapes could be restored rather precisely.

With one exception that would be discussed properly hereinafter, I identified four Hayes shapes and three forms based on the illustration presented by Zhuravlev that may be grouped in two functional categories: eating and drinking ware.

Eating ware is represented by plates of which Hayes $I^{20}$ are most numerous (pl. 1/1-3, 6). They have vertical slightly concave outside wall, bounded above and below on the outside by beveled mouldings, vertical thin, rounded rim delimited or not from the wall by fine grooves; almost flat floor and slightly concave on the inside, or deepened; beveled ring, short or tall (1-7). Fragments $8-25$, broken in the majority of cases immediately over the moulding from the lower part of the wall, with similar fabric and slip to fragments $1-7$, come most probably from Hayes I plates. Most analogies for this shape come from sites in the north of Pontus ${ }^{21}$ and also from Histria $^{22}$.

Fragments 26 and 27 (pl. 1/26), come from a plate type characterized by curving wall concave on the inside, beveled downturned rim, slightly concave floor on the inside and the junction between wall and floor marked with a deep groove on the inside and a fine groove on the outside. This shape is illustrated by Zhuravlev among the shapes which he considered distinctive for the Pontic sigillata A typological series ${ }^{23}$.

Pieces 28-31 (pl. 1/28) are the exceptional case I mentioned above. The shape as such curved walls, slightly vertical rim or strongly beveled on the inside, convex floor on the inside, with flat resting surface and junction between floor and wall marked with a groove on the inside - does not appear either in Hayes typology or the shapes illustrated by Zhuravlev, although the fabric and slip are similar to those of the pieces 6-7, 14, 171, definitely Pontic sigillata shapes, allow their inclusion in the same pottery class.

Drinking ware is slightly more varied topologically:

Hayes $V^{24}$ cups $(32-35, p l .2)$, conical body; rounded slightly inncurved rim with one moulding below or bounded above and below by mouldings on the outside; with the outside concave wall rises directly from the lines of the foot or outside concave wall and almost flat floor with groove inside at the jonction between rim and wall; and flat resting surface or beveled ring. Closest analogies for such pieces are signalled again in the north of Pontus ${ }^{25}$ and Histria ${ }^{26}$.

\footnotetext{
${ }^{19}$ Regarding the site reports and wandering of the archaeological material see Vulpe, Teodor 2003, 7-9, 13-14.

${ }^{20}$ Atlante II, 93, tav. XXII/6, 8.

${ }^{21}$ Khrschanovskiy 1998, grave 53, p. 77, table 1/1.1, grave 131-132, p. 84-85, table 3/3.1, 3.2; Zhuravlev 2002, fig. $121-2,5-6,8$.

${ }^{22}$ Suceveanu 2000, type XVII, 1-3, p. 63-67, pl. 23/1-3, 25/30.

${ }^{23}$ Zhuravlev 2002, fig. 12/3-4.

${ }^{24}$ Atlante II, 94, tav. XXIII/6, 9.

${ }^{25}$ Khrschanovskiy 1998 , graves $58,108,168$, p. $78,81,85$, table. $1 / 1.3,2 / 2.4,3 / 3.5$.

${ }^{26}$ Suceveanu 2000 , type XVII/5, p. 64, pl. 23/5; type XVIII/24, p. 73 , pl. $27 / 24$.
} 
Hayes VIII ${ }^{27}$ cups (36-39, pl. 2/36-37), bi-truncated cone body and concave slightly inncurved beveled rim bounded above and below on the outside by beveled mouldings, separated from wall by grooves; concave base, inside; wall meets floor at an outside moulding and an inside fine groove. As shown by similar pieces identified in north Pontic sites ${ }^{28}$ or at Histria $^{29}$, they have moulded foot.

Kantharoi Hayes $X^{30}$ (pl. 2/40-41, pl.3/42, 46) identified under both variants: bitruncated cone body, angular in the junction area between wall and floor $(40-47,82)$; or bitruncated cone body, rounded in the area of the maximum diameter $(48-58,65)$. Both variants exhibit vertical slightly inner wall with rounded rim, rounded base, flat floor, beveled ring and handle plate.

Fragments 58-64, 66-81, 83-92 may come from kantahroi belonging to any of the two variants described above. This shape has analogies in the north of Pontus as well ${ }^{31}$.

Globular cups identified in three variants: globular body, rounded rim, vertical or slightly outturned and junction between rim and wall marked with a fine groove on the outside (93-101, 103-113, 115-159, 179-182, pl. 3/93,150); globular body more flattened, rounded outturned rim, and the junction between wall and rim marked by fine grooves on the outside $(102,114, \mathrm{pl} .3 / 114)$; rounded rim, vertical or slightly outturned and globular beveled body (160 - 161, fig. 3/161). The three variants are characterised by rounded handles attached in the upper half of the body, concave floor with or without nipple on the inside and fine beveled ring. Fragments 162-178 may come from any of the three variants. This shape is illustrated by Zhuravlev among those which he considered typical for the Pontic sigillata A typological series $^{32}$.

"Collar" cups (183-184, pl.3/183) with rounded rim, straight; "collar" concave on the inside, globular body rounded in the area of the maximum diameter, concave floor, short beveled ring and two rounded handles, slightly heightened and attached to the body immediately under the collar. This shape is also illustrated by Zhuravlev among those which he deemed characteristic to Pontic sigillata $A^{33}$.

The fabric of the pieces discovered in the settlement from Poiana may be micaceous comprising mica in its composition; hard, porous with voids, white inclusions and occasionally with fine sand inside; or hard, vitreous alike fragments $(6-7,14,28,29,171$,). The fabric colour is generally red, reddish yellow or brown of various shades Munsell 5YR 7/4-6/3, 7.5YR 8/4, 5/6, 10YR 8/8, 8/6 and rarely Munsell 2.5 YR 7/4-7/8, 6/2, or 7.5 YR 6/1, 5/8.

The slip may be fine with metallic shiny or only flashy, occasionally with pearly patina; or thick, dull or shiny matte. Generally, the slip covers the vessels on the inside - in the plates' case - and on the outside up to over the ring, and in certain cases, like the globular cups or kantharoi Hayes X, it covers the vessel on the outside up to over the ring and on the inside only in the upper part. When covering the full vessel, the slip is much diluted in the area over the ring. Occasionally, the slip may be applied onto the vessel with the aid of a brush, case when it is rather thick, at times polished and preserves traces of the brush used for application. The slip colour varies alike clay in the same chromatic Munsell range, from red to brown, being regularly with a tone darker than the fabric. Exception makes the slip of pieces 28, 29 which is

\footnotetext{
${ }^{27}$ Atlante II, 94, tav. XXIII/9.

${ }^{28}$ Zhuravlev 2002, 259, fig. 15/7.

${ }^{29}$ Suceveanu 2000, type I/1, p. 10-11, pl. 1/1.

${ }^{30}$ Atlante II, 94-95, tav. XXIII/10-11.

${ }^{31}$ Zhuravlev 2002, fig. 15/1-2.

${ }^{32}$ Zhuravlev 2002, fig. 12/9, $15 / 4$.

${ }^{33}$ Zhuravlev 2002, fig. 12/11.
} 
reddish yellow on the rim Munsell 7.5 YR 6/8 and very dark brown in the rest Munsell 7.5 YR 2.5/2; and fragment 171 which is very dark brown Munsell 7.5 YR 2.5/2 onto the body and reddish yellow over the ring Munsell 7.5 YR 6/8.

The association of particular fabric types with certain slip types does not seem to follow a rule and likewise, vessels made of good quality fabric do not necessarily exhibit corresponding slip or the opposite. The best example is piece no. 1 whose fabric is hard, porous including several voids, mica and fine sand within the composition and is covered rather carefully with a fine, compact and shiny slip.

The decoration of the pieces identified at Poiana is specific to Pontic sigillata: rouletting - simple, double or triple strips - onto the base, inside the plates $(2,6,8,11,16-19$, 21-54, pl. 1/2,6) or the wall median part like the case of cup 37 (pl. 2/37); simple or double incised circles on fragments 1 and 25 (pl. 1/1); barbotine: vegetal or geometric motifs in the case of Hayes X kantharos (pl. 2/40-41, pl. 3/42, 65); vegetal motifs on the Hayes VIII $(36,38$ 39, pl. 2//36) cups' wall; "drops" (93-142, pl. 3/93, 114), "wheat grains" (143-148, pl. 3/146) or "leaves and drops" (pl. 3/149) like the case of globular cups. Many of them are also smooth (150-161, 169, 171, pl. 3/150).

We could identify a "planta pedis" stamp, type specific to Pontic sigillata, in a single case, inside plate no. $l$ and it was surrounded by two fine incised circles (pl.1/1).

Following chart 1 and chart 2 one may notice that functionally, drinking ware predominate (93\%) and globular cups are most numerous (49\%). Compared to other pottery categories of the Roman period, Pontic sigillata represent noticeably the lot best represented in the settlement from Poiana (chart 3).

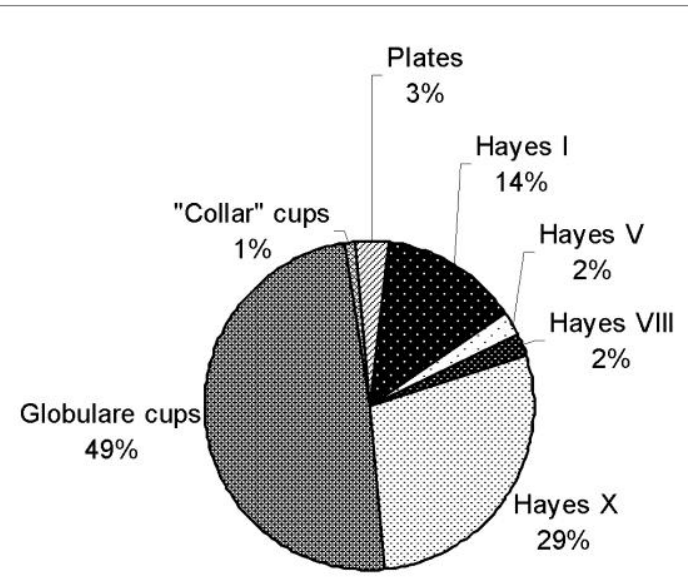

Chart 1: Ratio between pontic sigillata forms identified at Poiana 


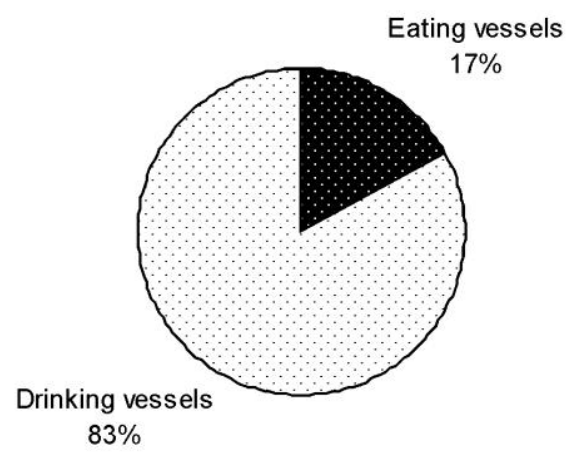

Chart 2: Ratio between pontic sigillata functional categories from Poiana

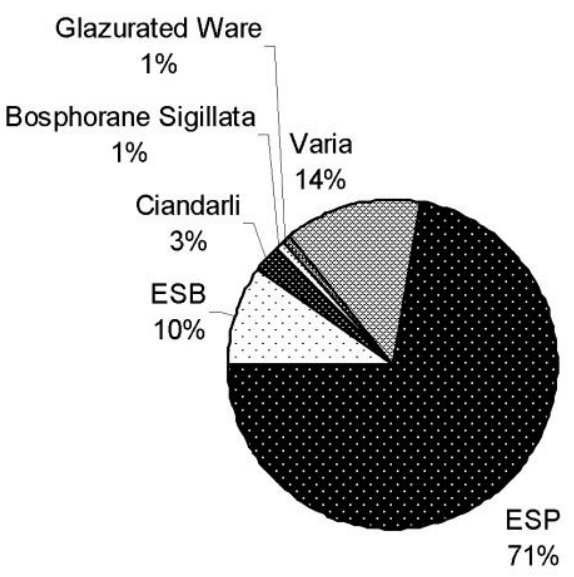

Chart 3. Ratio between roman pottery categories identified at Poiana

As mentioned by the beginning of the article, the simple reference to the items context of discovery does not allow chronological refinements concerning the emergence and use period of the Pontic sigillata in the settlement at Poiana and implicitly considerations regarding the controversial final date of the settlement ${ }^{34}$. Unfortunately not even D. Zhuravlev`s study, the most recent we could review, does not provide precise data on the pieces it illustrates as part of Pontic sigillata A typological series, mentioning only the period of their maximum diffusion second half of the $1^{\text {st }} \mathrm{c}$ to the first half of the $2^{\text {nd }} \mathrm{c} . \mathrm{AD}^{35}$. The exemplars from Histria similar to those at Poiana are also dated largely in the $1^{\text {st }}-2^{\text {nd }} c . \mathrm{AD}^{36}$. Only the items uncovered in the

\footnotetext{
${ }^{34}$ See Teodor, Vulpe, 20-22, 103-107, with bibliography.

${ }^{35}$ Zhuravlev 2002, 259-260.

${ }^{36}$ Suceveanu 2000, 64, 67, 73.
} 
barrow cemetery are dated closely: a Hayes I plate dated in the second half of the $1^{\text {st }}$ century AD and a Hayes VIII cup dated in the first half of the $1^{\text {st }} \mathrm{c} . \mathrm{AD}^{37}$.

In order to further detail, we took in consideration only the dating proposed for Hayes shapes. Thus, the kantharoi Hayes $\mathrm{X}$ could have emerged in the settlement as early as the beginning of the $1^{\text {st }} \mathrm{c}$. $\mathrm{AD}$, the shape being dated in the first half or mid (?) $1^{\text {st }} \mathrm{c}$. $\mathrm{AD}^{38}$, while Hayes I plates and Hayes V and VIII cups by mid century (Hayes I plates being dated from the second half of the $1^{\text {st }} \mathrm{c}$. AD until the $2^{\text {nd }} \mathrm{c}$. AD and Hayes V and VIII cups by mid $1^{\text {st }} \mathrm{c}$. AD deemed as the date when their production is initiated) ${ }^{39}$.

Generally, the Pontic sigillata were discovered in the last inhabitancy level of the settlement at Poiana - a level dated from the beginning of the $1^{\text {st }} \mathrm{c} . \mathrm{BC}$ and until mid $2^{\text {nd }}$ century $\mathrm{AD}^{40}$ - and reached the area only in the ${ }^{1 \text { st }}$ century $\mathrm{AD}$. It seemed rather interesting that we could not identify in the lot of analysed items shapes specific to the typological series Pontic sigillata B that start production from mid ${ }^{2 \text { nd }} \mathrm{c}$. AD.

\section{CATALOGUE}

1. MT, inv. no. 1224 (inv. no. 3553); h. 3cm; db. 16cm; df. 6cm; pl.1/1.

Fragment (half preserved; profile almost entire, ring poorly preserved). Vertical slightly concave outside wall, bounded above and below on the outside by beveled mouldings; vertical thin, rounded rim. Groove on the outside of wall and internal. Angular junction between wall and floor. Two circles incised and planta pedis stamp on the floor. Porous sandy fabric with many voids, reddish yellow Munsell 7.5YR 7/6. Reddish-yellow slip poorly preserved, covered whole inside and upper body outside, Munsell 5 YR 6/8.

Ref: Teodor 1994, p. 81, cat. 11, fig. 18; Vulpe, Teodor 2003, p. 323, cat. 804, fig. $235 / 8$.

2. MT; 1951, L.; pl.1/2.

Restored (profile entire). Vertical slightly curved outside wall, bounded above and below on the outside by beveled mouldings; vertical, rounded rim; rounded floor; rounded junction between wall and floor.

Ref: Teodor 1994, p. 80, cat. 5, fig. 11/1; Vulpe, Teodor 2003, p. 323, cat. 798, fig. 235/9; Note: drawing and description after Vulpe, Teodor 2003, p. 323, cat. 798, fig. 235/9.

3. MT; P 50, K 4, db. 17cm; pl. 1/3.

Fragment of vertical slightly inner wall bounded on the outside by two beveled mouldings; fine, rounded rim. Homogenous hard reddish-yellow fabric, Munsell 5YR 7/6. Fine flaky slip, reddish-yellow Munsell 5 YR 7/6.

4. MT; P. 50, K 3, db. $20 \mathrm{~cm}$.

Fragment of vertical slightly inner wall bounded on the outside by two beveled mouldings; vertical fine, rounded rim. Homogenous hard reddish-yellow fabric, Munsell 5 YR 7/6. Fine flaky slip, reddish-yellow Munsell 5 YR 7/6.

5. MT ; Poiana, 50; J, db. $12 \mathrm{~cm}$.

Fragment of vertical slightly inner wall, bounded on the outside by two beveled mouldings; vertical fine rounded rim. Hard porous fabric with voids and mica, reddish yellow Munsell 5YR 7/6. Compact thick polished slip covered whole inside and upper body outside, trickled on the base, reddish yellow, Munsell 5YR 6/8.

\footnotetext{
${ }^{37}$ Suceveanu 2000, type XVII, 1, p. 63; type I/1, p. 10-11.

${ }^{38}$ Atlante II, 94-95.

${ }^{39}$ Atlante II, 93, 94.

${ }^{40}$ Vulpe, Teodor 2003, 20.
} 
6. MT; P.; db. 18cm; pl.1/6.

Fragment (one quarter preserved), almost entire profile, waste preserved ring. Vertical slightly everted wall, bounded on the outside by two beveled mouldings; fine rounded slightly everted rim; rounded floor and concave inside; angular junction between wall and floor with inside fine groove; low beveled ring. Fine groove on the outside wall, rouletting on inside base. Vitreous hard light grey fabric Munsell 7.5 YR 6/1; shiny strong brown slip Munsell 7.5 YR 5/8.

7. MT; P.; db. $16 \mathrm{~cm}$.

Fragment (one quarter from wall and floor preserved). Vertical slightly outer wall, bounded on the outside by two beveled mouldings; fine rounded slightly outturned rim; rounded floor and concave inside; angular junction between wall and floor with fine groove, inside; low beveled foot-ring. Fine groove on the outside wall. Vitreous hard reddish yellow fabric Munsell 7.5 YR 8/6; shiny reddish yellow slip Munsell 7.5 YR 7/8.

8. MT; P 88 O $\uparrow$ V $/ 5$ sch. 2 ; df. $10 \mathrm{~cm}$.

Three wall and floor fragments. Vertical wall, fragmentary beveled moulding on the outside; concave slightly rounded floor; beveled foot-ring. Rouletting: three double circles on innerface of floor. Porous fabric with mica and voids inside, reddish yellow Munsell 7.5YR 7/6; dull slip cover the whole inside and upper body outside, reddish yellow Munsell 7.5 YR 7/8.

9. MT; fs. $3.5 \times 2.5 \mathrm{~cm}$.

Wall and floor small fragment. Vertical wall with fragmentary beveled moulding. Hard reddish yellow fabric with voids and white inclusions, Munsell 5YR 7/8; shiny flaky reddishyellow slip, Munsell 5 YR 6/8.

10. MT; P.; fs. 5.2 x $4 \mathrm{~cm}$.

Small fragment. Vertical wall with fragmentary beveled moulding. Fine reddish yellow fabric, Munsell 5 YR 7/8; shiny reddish yellow slip, compact on the inside and on the upper body, diluted on the floor, outside, Munsell 5YR 6/8.

11. MT; P. 50, J 4; fs. $5.3 \times 3.6 \mathrm{~cm}$.

Fragment of flat floor; fragmentary beveled moulding on the outside; rouletting on the inside. Porous hard fabrics with voids, reddish yellow, Munsell 7.5YR 8/6; homogeneous shiny slip on the inside, missing on base, reddish yellow Munsell 7.5 YR 7/8.

12. MT; P 50, J 3; fs. $8.7 \times 6.7 \mathrm{~cm}$.

Flat floor small fragment; fragmentary beveled moulding on the outside. Fine homogeneous fabric, reddish yellow Munsell 7.5 YR 8/6; washy slip on the inside; remains on the outside, reddish yellow Munsell 7.5 YR 7/8.

13. MT; P 50, J c 3-4, 1m; fs. 8.4 x $9.6 \mathrm{~cm}$.

Flat floor small fragment; fragmentary beveled moulding on the outside. Hard porous fabric with voids and white inclusions, reddish yellow Munsell 7.5 YR 7/6. Thick shiny slip inside, dull diluted outside, reddish yellow Munsell 7.5 YR 7/8.

14. MT; P 50, J c 3-4, $-1 \mathrm{~m}$; fs. 10.8 x 8.2cm.

Flat floor small fragment; fragmentary beveled moulding on the outside. Hard compact vitreous fabric, similar with 7 and 6, reddish yellow, Munsell 7.5 YR 8/6; fine adherent shiny slip, reddish yellow, Munsell 7.5 YR 7/8.

15. MT; P; fs. 6.1 x $2.9 \mathrm{~cm}$.

Flat floor small fragment; fragmentary beveled moulding on the outside. Reddish yellow hard fabric with mica Munsell 5 YR 7/6. Fine compact shiny slip on the inside, diluted on the outside, reddish yellow Munsell 5YR7/8.

16. MT; P 50, L 5; fs. 5.8 x $3.3 \mathrm{~cm}$. 
Flat floor small fragment; fragmentary beveled moulding on the outside; rouletting on the inside, fine groove on the outside. Reddish yellow porous fabric, Munsell 5 YR 7/8; fine shiny reddish yellow slip, Munsell 7.5 YR 7/8.

17. MT; P. 50 , L3; fs. $5 \times 2.5 \mathrm{~cm}$.

Floor fragment; fine narrow beveled ring; rouletting on the floor. Hard porous fabric with voids and white inclusions, reddish yellow Munsell 7.5 YR 7/6; fine shiny slip inside only, reddish yellow Munsell 7.5 YR 7/8.

18. MT; P. $86 \mathrm{~N}, \uparrow 26,-3.50 \mathrm{~m}$; fs. $5 \mathrm{x} 4 \mathrm{~cm}$.

Floor fragment; rouletting on the floor. Porous reddish yellow fabric, Munsell 7.5YR 7/6; dull reddish yellow slip inside, patches on the outside, Munsell 7.5YR 7/8.

19. MT; P.; fs. $4.2 \times 3.8 \mathrm{~cm}$.

Floor fragment; rouletting on the floor; low beveled ring with groove on the flat resting surface. Porous reddish yellow fabric with voids, Munsell 5YR 7/6; fine shiny slip inside, patches on the outside, reddish yellow, Munsell 5 YR 7/8.

20. MT; P.; df. $6 \mathrm{~cm}$.

Floor fragment; beveled ring with flat resting surface; groove on the innerface of floor. Compact hard fabric with white inclusions, reddish yellow Munsell 5YR 7/8; compact shiny slip inside only, reddish yellow Munsell 5YR 6/8.

21. MT; P. 50, J4; fs. 5.4 x $2 \mathrm{~cm}$.

Floor fragment; beveled ring with flat resting surface; rouletting on the innerface of floor. Micaceous fabric, reddish yellow, Munsell 7.5YR 8/6; patches of reddish yellow slip Munsell 7.5YR 7/8.

22. MT, inv. no. 3531 ; df. $8 \mathrm{~cm}$.

Floor fragment; fragmentary beveled mouldings from the bottom of wall; beveled ring foot; rouletting on the innerface of floor. Fine reddish yellow fabric, Munsell 5YR 7/6; flaky dull slip, reddish yellow Munsell 5YR 6/8.

23. MT; P. 50, Lc. 4 ; df. $7 \mathrm{~cm}$.

Floor fragment; fine beveled ring; rouletting on the innerface of floor. Hard fine reddish yellow fabric with voids, Munsell 5 YR 7/6; compact fine shiny slip, reddish yellow, Munsell 5 YR 6/8.

24. MT; P. 50, J², $-2 \mathrm{~m}$; df. $7 \mathrm{~cm}$.

Floor fragment; fragmentary beveled moulding on the outside; low beveled ring; rouletting on the innerface of floor. Reddish yellow fabric, Munsell 5YR 7/6; fine reddish yellow slip, shiny to metallic, Munsell 5YR 7/8.

25. MT ; P 50 L 2; fs. 5 x $3.5 \mathrm{~cm}$.

Floor fragment; low ring; shallow incised circle on the innerface of floor. Hard fabric with voids and white inclusions, reddish yellow, Munsell 5YR 6/6; fine adherent slip, inside only, yellow red Munsell 5YR 5/8.

26. MT, inv. no. 3536; db. $26 \mathrm{~cm}$; h $4.2 \mathrm{~cm}$; pl.1/26.

Fragment (half preserved; floor missing). Beveled downturned rim; curving wall; rounded junction between wall and floor with grooves inside and outside. Hard compact light grey fabric, Munsell 10YR 7/1; fine shiny slip cover the whole inside and upper body outside only, yellow, Munsell 10YR 7/8.

27. MT; P 50, j5; db. $21 \mathrm{~cm}$.

Fragment (1/2 preserved; floor missing). Beveled downturned rim; curving wall; rounded junction between wall and floor with grooves inside and outside. Hard compact reddish 
yellow Munsell 5YR 7/6; fine shiny slip cover the whole inside and upper body outside only, reddish yellow Munsell 5 YR 6/8.

28. MT; P. 1951, L 9/3; db. 30cm; fig. 1/28.

Fragment (one quarter preserved; floor missing). Vertical inner beveled rim; curving wall; almost flat slightly concave outside floor; groove by the junction between wall and floor, inside. Hard vitreous fabric, similar with 6-7 and 18, reddish yellow, Munsell 7.5YR 7/6; fine shiny slip covered the whole inside and upper body outside only, trickle on the outside floor, very dark brown, Munsell 7.5 YR 2.5/2 and reddish yellow on the rim Munsell 7.5 YR 6/8.

29. MT; P.; fs. 5 x $4 \mathrm{~cm}$.

Fragment. Vertical rim, inner beveled; curving wall; Hard glassily fabric, similar with 6-7, 18 and 28, reddish yellow, Munsell 7.5YR 7/6; fine shiny slip, very dark brown Munsell 7.5YR 2.5/2, reddish yellow on the rim, Munsell 7.5YR 6/8.

30. MT; P. $50 \mathrm{~L}$; db. $14 \mathrm{~cm}$.

Fragment. Vertical rim, inner beveled; curving wall; almost flat slightly concave outside floor; groove at junction between wall and floor, inside. Porous fabric with mica, reddish yellow, Munsell 7.5YR7/6; flaky thick slip, covered the whole inside and upper body only, outside, reddish yellow, Munsell 7.5YR7/8.

31. MT; P. 50, L, c4; db. $16 \mathrm{~cm}$.

Fragment. Vertical rim, inner beveled; curving wall; almost flat slightly concave outside floor; groove at junction between wall and floor, inside. Porous fabric with mica, pink, Munsell 7.5YR 7/3; flaky thick shiny slip, covered the whole inside and upper body only, outside, reddish yellow, Munsell 7.5YR7/8.

32. MT, inv. no. $6285 ; \mathrm{db} .11 \mathrm{~cm}$; df. $4.8 \mathrm{~cm}$; h $5.2 \mathrm{~cm}$; pl.2/32.

Restored (three-quarter preserved; profile almost entire). Conical body; rounded slightly inncurved rim with one moulding below on the outside; on the exterior, the outside concave wall rises directly from the lines of the foot; foot concave outside; flat resting surface. Porous hard fabric with abound mica, reddish yellow, Munsell 7.5 YR 7/8; thick adherent dull slip, cover the whole inside, trickle on the outside, reddish yellow, Munsell 5 YR 6/8.

Ref: Vulpe, Teodor, 2003, p. 325, cat. 822, fig. 237/11.

33. MT; P 50 K' Podina I; db. 10cm; h. 5.2cm; pl.2/33.

Fragment (one-quarter rim and wall preserved). Conical body and concave slightly inncurved rim bounded above and below on the outside by mouldings, separated from wall by grooves. Groove inside at the junction between rim and wall. Hard compact fabric, reddish yellow, Munsell 7.5 YR 7/6; fine adherent shiny slip, reddish yellow with black patches, Munsell 7.5 YR 6/6.

34. MT, inv. no. 2278; P. 87, N1, $-0.75 \mathrm{~m} ;$ db. $12 \mathrm{~cm}$; h. $3.6 \mathrm{~cm} ;$ pl.2/34.

Fragment (one quarter rim and wall preserved). Conical body and concave slightly inncurved rim bounded above and below on the outside by beveled mouldings, separated from wall by grooves; groove inside at the junction between rim and wall. Hard fabric, with white inclusions, reddish yellow; thick adherent dull slip, reddish yellow, Munsell 5 YR 6/8.

Ref: Vulpe, Teodor 2003, p. 325, cat. 821, fig. 237/10.

35. MT, inv. no. 3015; P. 87, N1, 1 m 19, -0.75 m; db. 11, df. 5.8cm, h. 5.3cm; pl. 2/35.

Fragment (two thirds preserved, floor missing). Conical body and concave slightly inner rim bounded above and below on the outside by beveled mouldings, separated from wall by grooves; groove inside at the junction between rim and wall; beveled ring with resting surface flat. Homogenous fabric, reddish yellow, Munsell 5YR 7/6; flaky fine shiny slip, cover the whole inside and outside upper the body, above the ring, reddish yellow, Munsell 5YR 7/8. 
Ref: Vulpe, Teodor 2003, p. 325, cat. 818, fig. $237 / 7$.

36. MT, inv. no. 1653. P. 1926, section A; fs. 10 x 10cm; pl.3/36.

Fragment (one quarter wall preserved). Vertical wall; vertical thickened outside rim bounded above and below by mouldings, separated from wall by grooves; concave base, inside; the wall meets base at an outside moulding and an inside fine groove. Barbotine decoration on the outside wall; vegetal motifs. Homogeneous hard fabric, reddish yellow, Munsell 5YR 7/6; fine slip, cover the upper body only, trickled inside and base, outside, reddish yellow, Munsell 5YR $7 / 8$.

Ref: Vulpe, Teodor 2003, p. 328, cat. 846, fig. 239/3; Note: drawing after VulpeTeodor 2003, fig. 239/3.

37. MT; P. 1950, J², -2 ; db. $16 \mathrm{~cm}$; h. $16 \mathrm{~cm}$; fig. $3 / 37$.

Fragment (one-quarter wall preserved). Vertical wall; vertical slightly incurved beveled rim, concave on the outside, bounded above and below by mouldings separated from wall by grooves. Rouletting at the middle wall, outside. Homogeneous hard fine fabric, reddish yellow Munsell 5YR 7/6; fine reddish yellow slip, Munsell 5YR 6/8.

38. MT; P 50, J III-IV; fs. 5.8 x $9.4 \mathrm{~cm}$.

Fragment (one-quarter wall and base preserved). Vertical wall, concave base, inside; wall meets base at an outside beveled moulding. Barbotine decoration on the wall, outside. Hard homogeneous fabric with white inclusions, reddish yellow, Munsell 7.5YR 8/6; fine adherent metallic slip at the outside wall only, reddish yellow, Munsell 7.5YR 7/8.

39. MT; P; fs. $5.9 \times 5 \mathrm{~cm}$.

Fragment (one-quarter wall and base preserved). Vertical wall, concave base, inside; wall meets base at an outside beveled moulding. Barbotine decoration on the wall, outside. Porous fabric with voids and white inclusions, reddish yellow, Munsell 7.5YR; dull fine adherent slip cover upper body only, reddish yellow, Munsell 7.5YR 7/8.

40. MNA, inv. no. 636 ; db. $13 \mathrm{~cm}$; h. $6.3 \mathrm{~cm}$; fig. $3 / 40$.

Entire. Bi-truncated cone body, angular at the junction between wall and base; vertical slightly inner wall with rounded rim; rounded base; flat floor; beveled ring; handle plate; barbotine decoration on the upper body, outside; vegetal motifs.

Ref: Glodariu 1974, cat. 34/35, p.214, pl. XXIV; Note: description and drawing after Glodariu 1974, p. 214, cat. 34/35, pl. XXIV.

41. MT, inv. no. $2299, \mathrm{P} 86 \mathrm{~N},-1 \mathrm{~m}$; db. $10 \mathrm{~cm}$; h. $5.5 \mathrm{~cm} ; \mathrm{pl} .3 / 41$.

Fragment (half wall and one handle preserved). Bi-truncated cone body, angular at the junction between wall and base; vertical slightly inner wall with rounded rim; rounded base; flat floor; beveled ring; handle plate; barbotine decoration on the upper body. Homogenous sandy fabric, pink, Munsell 5YR 7/4; entire slip missing.

Ref: Teodor 1994, cat. 57, p. 92, fig. 5/8; Vulpe, Teodor 2003, cat 850, p. 328, fig. 239/8; Note: drawing after Vulpe, Teodor 2003, fig. 239/8.

42. MT, inv. no. 2298; P. 1985, S. N, $\uparrow \quad 19,-1.20 \mathrm{~m} ; \mathrm{N},-0.50 \mathrm{~m}$; db. $12 \mathrm{~cm}$; h $6.3 \mathrm{~cm}$; pl. $4 / 42$.

Fragment. Bi-truncated cone body, angular at the junction between wall and base; vertical slightly inner wall with rounded rim; rounded base; flat floor; beveled ring; handle plate; barbotine decoration on the upper body, outside. Compact hard fabric, reddish yellow Munsell 5YR 7/6; fine shiny slip cover the whole outside and inside upper body only, reddish yellow, Munsell 5YR6/8.

Ref: Teodor 1994, cat. 56, p. 92, fig. 5/6; Vulpe, Teodor 2003, cat. 848, p. 328, fig. 239/6; Note: drawing after Vulpe, Teodor 2003, fig. 239/6. 
43. MT, inv. no. 1186 ; P; fs. 4.4 x $3 \mathrm{~cm}$.

Fragment. Vertical wall; angular junction between wall and base; barbotine decoration; vegetal motifs. Homogeneous fine fabric, reddish yellow, Munsell 7.5YR 7/6; fine poorly preserved slip, strong brown, Munsell 7.5 YR 5/6.

44. MT; P 50, $\mathrm{J}^{2},-2 \mathrm{~cm}$; fs. $3 \times 5 \mathrm{~cm}$.

Fragment (one quarter preserved). Bi-truncated cone body, rounded at the junction between wall and base; rounded rim, vertical slightly inner wall; barbotine decoration. Porous hard fabric with white inclusions, reddish yellow, Munsell 7.5 YR 7/6; fine, shiny slip, reddish yellow, Munsell 7.5 YR 7/8.

45. MT; P 50, J 8; db. $10 \mathrm{~cm}$.

Fragment (one-quarter preserved). Bi-truncated cone body; rounded rim, vertical rounded slightly inner wall; barbotine decoration. Homogeneous fabric, reddish yellow, Munsell 5YR 7/6; fine shiny slip, reddish yellow, Munsell 5YR 6/8.

46. MT; P 50, L3, fs. $3.5 \times 3 \mathrm{~cm}$.

Small fragment of vertical slightly inner wall; wall meets floor at angle. Porous fabric with abundant mica, yellow Munsell 10 YR 8/8; slip entire waste.

47. MT, inv. no. 1027; P 49, H', II S, $-1,00 \mathrm{~m}$, no. 124; db. $12 \mathrm{~cm}$.

Fragment (one-quarter of wall preserved). Bi-truncated cone body angular at the junction between wall and floor; vertical rounded rim; vertical slightly inner wall; barbotine decoration. Fine reddish yellow fabric Munsell 5YR 7/8; fine slip outside, trickled inside, yellow red, Munsell 5 YR 5/6.

48. MT; P 50, HJ; fs. $2.3 \times 5.2 \mathrm{~cm}$.

Fragment (one-quarter of wall preserved). Vertical slightly inner wall; concave floor, inside; barbotine decoration, vegetal motifs. Compact fine reddish yellow fabric, Munsell 7.5YR 7/6; fine shiny slip, diluted on the floor, outside, reddish yellow Munsell 7.5 YR 6/8.

49. MT; P 50, I, VIII, -9 , db. $8 \mathrm{~cm}$.

Fragment (one-quarter of wall preserved). Bi-truncated cone body, rounded at the junction between wall and floor; vertical rounded rim, vertical rounded slightly inner wall; inside concave floor; handle plate. Porous fabric with mica and fine sand, reddish yellow, Munsell 7.5YR 7/6; fine shiny slip outside, on the upper body, inside reddish yellow, Munsell 7.5 YR $7 / 8$.

50. MT; P. 28, db. $8 \mathrm{~cm}$.

Fragment (one-quarter of wall preserved). Bi-truncated cone body, rounded at the junction between wall and floor; vertical rounded rim, vertical rounded slightly inner wall; inside concave floor. Compact hard light grey fabric, Munsell 5YR 7/1; shiny yellowish-red slip Munsell 5YR 5/8.

51. MT; P 51, L, O discontinued"; fs. $5.1 \times 4.2 \mathrm{~cm}$.

Fragment (one-quarter of wall preserved). Bi-truncated cone body, rounded at the junction between wall and floor; fine beveled ring. Homogeneous pink fabric, Munsell 7.5YR 7/4; fine shiny slip on the upper body, trickled on the floor, strong brown Munsell 7.5YR 5/8.

52. MT; fs. 6.4 x $3.4 \mathrm{~cm}$.

Fragment. Bi-truncated cone body, rounded at the junction between wall and floor; fine beveled ring. Homogeneous pink fabric, Munsell 7.5YR 7/4; fine shiny slip on the upper body, trickled on the floor, strong brown Munsell 7.5YR 5/8.

53. MT; P 50, K, c6; fs. $5.2 \times 3.5 \mathrm{~cm}$.

Fragment. Vertical slightly inner wall, rounded junction between wall and floor; concave floor inside; barbotine decoration, vegetal motive. Fine fabric with white inclusions, 
light red Munsell 2.5YR7/6; shiny red slip outside, dull inside, missing on the floor, outside, Munsell 2.5YR5/8.

54. MT; P 50; fs. 5.1 x $3 \mathrm{~cm}$.

Fragment. Vertical slightly inner wall, rounded junction between wall and floor; concave floor inside. Compact hard fabric, pinkish white, Munsell 5YR 8/2; fine shiny to metallic slip on the upper body, missing on the foot, yellowish red, Munsell 5YR 4/6.

55. MT; P.; fs. 3 x $3.5 \mathrm{~cm}$.

Fragment. Vertical slightly inner wall, rounded junction between wall and floor; concave floor inside. Compact hard fabric, pinkish white, Munsell 5YR 8/2; fine shiny to metallic slip on the upper body, missing on the foot, yellowish red, Munsell 5YR 4/6.

56. MT; P 50. K, c 6-8 (KM); fs. 4.7 x $3.2 \mathrm{~cm}$.

Fragment. Vertical slightly inner wall, concave floor inside. Homogeneous fine pink fabric, Munsell 5YR7/4; flaky shiny reddish yellow slip on the body, missing on the outside floor, Munsell 5YR 6/8.

57. MT; P 50, Lc.2; fs. 2.2 × $2.8 \mathrm{~cm}$.

Fragment. Vertical slightly inner wall, rounded junction between wall and floor; concave floor inside; barbotine decoration; vegetal motifs. Homogeneous fine reddish yellow fabric, Munsell 7.5YR7/6; thick dull reddish yellow slip, Munsell 7.5YR 7/8.

58. MT, inv. no. 2295; P 28; db. $10 \mathrm{~cm}$.

Fragment. Bi-truncated cone body, rounded at the junction between wall and floor; fine, vertical rim; vertical rounded wall; one handle plate; barbotine decoration. Compact fine reddish yellow fabric, Munsell 5YR 6/8; thick dull slip, yellowish-red, Munsell 5YR5/8.

Ref: Teodor 1994,cat. 51, p. 90, fig. 5/1; Vulpe, Teodor 2003, cat. 844, p. 237, fig. 239/1.

59. MT; P 50, Jc4; db. $12 \mathrm{~cm}$.

Fragment (one quarter preserved). Vertical slightly rounded rim; vertical wall; barbotine decoration, vegetal motive. Compact fine fabric reddish yellow, Munsell 5YR 7/4; flaky thick dull yellow red slip with black patches Munsell 5YR 5/6.

60. MT, inv. no. 1040; P 49, H', II S, -1.50 m, no. 258 ; db. 14 cm.

Small fragment of vertical wall; rounded vertical rim; barbotine decoration, vegetal motive. Fine reddish yellow fabric, Munsell 5YR 7/6; compact thick yellow red slip, Munsell 5YR 5/8.

61. MT; P 1951, $-0.90 \mathrm{~m} ; \mathrm{db} .8 .5 \mathrm{~cm}$.

Small fragment of vertical wall; rounded vertical rim; barbotine decoration, vegetal motifs. Compact fine reddish yellow fabric; reddish yellow slip, Munsell 7.5 YR 6/8.

62. MT; P 85, Ni 16, $1.60 \mathrm{~m}$; db. $14 \mathrm{~cm}$.

Small fragment of vertical wall; rounded vertical rim; one handle plate decorated with two darts; barbotine decoration on the outside wall. Compact hard strong brown fabric Munsell 7.5 YR 7/4; compact strong brown slip, Munsell 7.5 YR 7/6; brush traces, outside.

63. MT; P. 50, K, c 6-8; fs. 3.5 x 4.4 .

Small fragment of vertical wall; rounded vertical rim; barbotine decoration on the outside wall. Compact pink fabric, Munsell 5YR 7/4; thick dull slip, yellowish red, Munsell 5YR 5/6.

64. M.T.; P 90, P` 39, 1.30m; fs. 5.7 x $5.9 \mathrm{~cm}$.

Small fragment of vertical wall; rounded vertical rim; barbotine decoration on the outside wall. Compact reddish yellow fabric, Munsell 7.5YR 7/6; shiny yellowish red slip, Munsell 5YR 5/8.

65. MT; P 49, I N, -1.40 x 1.60m; db. $8 \mathrm{~cm}$, h. 4.8cm; pl. 4/65. 
Small fragment of vertical wall; rounded vertical rim; barbotine decoration, vegetal motifs. Homogeneous light brown fabric, Munsell7.5 YR 6/3; dull very dark brown slip, Munsell 7.5 YR 2.5/2.

66. MT; P. 50, I and I'; db. $8 \mathrm{~cm}$.

Small fragment of vertical wall; rounded vertical rim; barbotine decoration, vegetal motifs; one handle plate. Homogeneous reddish yellow fabric, Munsell 7.5 YR 7/6; fine yellowish red slip, Munsell 5 YR5/8.

67. MT; P. 30 E, $1.40 \mathrm{~m} ; \mathrm{db} .10 \mathrm{~cm}$.

Fragment. Rounded vertical rim, one handle plate. Homogeneous fabric with voids, pale red, Munsell 2.5YR 6/2; poorly preserved fine slip, dark red Munsell 2.5YR 3/6.

68. MT; P.; fs. 2.5 x $3 \mathrm{~cm}$.

Fragment. Rounded vertical rim, one handle plate. Homogeneous fabric with voids, pale red, Munsell 2.5YR 6/2; poorly preserved fine slip, dark red Munsell 2.5YR 3/6.

69. MT; P 50, J5; fs. $3.6 \times 3.5 \mathrm{~cm}$.

Fragment of wall; barbotine decoration. Homogeneous fine fabric, pink Munsell 7.5YR 7/3; dull fine slip, reddish yellow, Munsell 7.5YR7/8.

70. MT; P.; fs. $2.6 \times 3 \mathrm{~cm}$.

Fragment. Rounded vertical rim; one handle plate. Hard fabric with white inclusions pink, Munsell 5YR 7/4; thick dull slip, yellowish red Munsell 5YR 5/8.

71. MT; P 50, K 21; fs. 3.5 x $2.9 \mathrm{~cm}$.

Wall fragment; barbotine decoration. Homogeneous light red fabric, Munsell 2.5YR 7/6; homogeneous dull red slip Munsell 2.5YR 5/5.

72. MT; P 88, O; db. 8cm.

Fragment. Vertical wall; vertical rounded rim; one handle plate decorated with dart. Fine reddish yellow fabric, Munsell 7.5YR 7/6; thick dull reddish yellow slip, Munsell 7.5YR 6/8.

73. MT; P 1951, sondage Apreotesei; fs. $3.6 \times 3.2 \mathrm{~cm}$.

Wall fragment; barbotine decoration, vegetal motifs. Homogeneous fine light red fabric Munsell 2.5 YR 7/6; flaky light red slip, Munsell 2.5 YR 6/8.

Ref: Vulpe, Teodor 2003, cat. 861, p. 329, fig. 240/11.

74. MT; P 50, J 5; fs. 4.6 x $2.9 \mathrm{~cm}$.

Wall fragment; barbotine decoration; vegetal motifs. Homogeneous light red fabric, Munsell 2.5YR 7/6; fine dull red slip Munsell 2.5 YR 4/6.

75. MT; P 50, L c; db. $8 \mathrm{~cm}$.

Vertical wall fragment; rounded slightly outturned rim; barbotine decoration. Homogeneous reddish yellow fabric, Munsell 7.5YR7/6; thick dull reddish yellow slip, Munsell 7.5YR 7/8, poorly preserved.

76. MT; P. 50, L, c4, fs. $2 \times 4 \mathrm{~cm}$.

Vertical wall fragment; rounded slightly outer rim; barbotine decoration. Hard light reddish brown fabric, Munsell 2.5YR7/4; thick shiny slip, on the outside upper body, only, light red, Munsell 2.5YR 6/8.

77. MT; P 50, k4; fs. 4.2 x $3.2 \mathrm{~cm}$.

Wall fragment; barbotine decoration. Homogeneous light reddish brown fabric, Munsell 2.5YR 7/4; thick dull red slip, Munsell 2.5YR 5/8.

78. MT; P. 50, L; db. 8 cm.

Vertical wall fragment; vertical rounded rim; one handle plate. Homogeneous grey fabric, Munsell 2.5Y 6/1; dull light olive brown slip, Munsell 2.5Y 5/3. 
79. MT; fs. $3.4 \times 2.2 \mathrm{~cm}$.

Vertical wall fragment; vertical rounded rim; one handle plate. Homogeneous reddish yellow fabric, Munsell 5YR 7/6; dull reddish yellow slip, Munsell 5YR 6/8.

80. MT; P 50; fs. $2.1 \times 2.9 \mathrm{~cm}$.

Vertical wall fragment; vertical rounded rim; barbotine decoration. Homogeneous light reddish brown fabric, Munsell 2.5YR 7/4; thick dull red slip, Munsell 2.5YR 5/8.

81. MT; P 50, K c 7; fs. 2.8 x $2.8 \mathrm{~cm}$.

Vertical wall fragment; vertical rounded rim; barbotine decoration. Homogeneous light reddish brown fabric, Munsell 2.5YR 7/4; thick dull red slip, Munsell 2.5YR 5/8.

82. MT; P 5 c L 10/42, c3; fs. $2.5 \times 2.9 \mathrm{~cm}$.

Vertical wall fragment; angular junction between wall and floor; barbotine decoration; vegetal motifs. Hard fine light red fabric, Munsell 2.5YR7/6; thick shiny red slip, Munsell 2.5 YR 5/8.

83. MT; P 50, L 4; fs. 3.5 x $3 \mathrm{~cm}$.

Vertical wall, rounded slightly outer rim; barbotine decoration. Homogeneous reddish yellow fabric, Munsell 7.5YR7/6; thick dull reddish yellow slip, Munsell 7.5YR 7/8.

84. MT; P 50, 1, c5; fs. 1.3 x $2 \mathrm{~cm}$.

Wall fragment; barbotine decoration. Homogeneous reddish yellow fabric, Munsell 7.5YR7/6; thick dull reddish yellow slip, Munsell 7.5YR 7/8.

85. MT; P. 50, k 7; fs. 2.6 x $2.7 \mathrm{~cm}$.

Wall fragment, slightly rounded; barbotine decoration. Homogeneous reddish yellow fabric, Munsell 7.5YR7/6; reddish yellow slip, poorly preserved, Munsell 7.5YR7/8.

86. MT; P. 50, L, c 5; fs. 3.3 x $2.2 \mathrm{~cm}$.

Vertical wall fragment; barbotine decoration. Homogeneous light reddish brown fabric, Munsell 2.5YR 7/4; thick dull red slip, Munsell 2.5YR 5/8.

87. MT; P 50, J 10, db. $8 \mathrm{~cm}$.

Vertical wall fragment; vertical rounded rim; one handle plate. Homogeneous pink fabric, Munsell 7.5YR7/3; fine shiny slip; brush traces, reddish yellow Munsell 7.5YR 7/8.

88. MT; P. 50, L, J I; df. 6 cm.

Three floor fragment; fine beveled ring. Reddish yellow fabric, Munsell 5YR 7/6; compact dull yellow red slip, washy outside, Munsell 5YR 5/8.

89. MT; P 87, N; fs. 3.6 x $3 \mathrm{~cm}$.

Vertical wall fragment; barbotine decoration. Homogeneous fine light red slip, Munsell 2.5YR 7/6; thick dull light red slip, Munsell 2.5YR 7/8.

90. MT; P 50, L J III; fs. 2.4 x $1,8 \mathrm{~cm}$.

Vertical wall, rounded slightly outturned rim; barbotine decoration. Homogeneous fine pink slip, Munsell 2.5YR7/4; thick dull red slip Munsell 2.5YR4/8.

91. MT; P 50 K 6; fs. 4 x $3.3 \mathrm{~cm}$.

Vertical slightly rounded wall; rounded rim; one handle plate. Homogeneous fabric with voids, pale red, Munsell 2.5YR 6/2; dark red slip, poorly preserved, Munsell 2.5YR 3/6.

92. MT; P. $30, \mathrm{E},-25$, fs. 4 x $1.5 \mathrm{~cm}$.

Vertical slightly rounded wall; rounded rim; one handle plate. Homogeneous fabric with voids, pale red, Munsell 2.5YR 6/2; dark red slip, poorly preserved, Munsell 2.5YR 3/6.

93. MT, inv. no. 6287, old collection; db $8 \mathrm{~cm}$, df. $3.8 \mathrm{~cm}$; pl. 4/93.

Restored (three quarter preserved, entire profile). Globular body; fine thickened rounded outturned rim; rounded handle; fine groove at the junction between rim and wall; concave floor, inside; beveled ring with flat resting surface; barbotine decoration, "drops" on 
the superior part of body. Hard fine fabric with white inclusions, reddish yellow, Munsell 7.5YR 7/6; fine shiny slip, poorly preserved, on the outside upper body only, reddish yellow, Munsell 7.5YR 7/8.

Ref: Glodariu 1974, c34/50, p. 214, pl. XXIV; Teodor 1994, cat.56, p. 91, fig. 5/7; Vulpe, Teodor 2003, cat. 849, p. 328, fig. 239/7; Note:drawing after Glodariu 1074, pl. $\mathrm{XXIV/c34/50.}$

94. MT; P50 L3; fs. $2.5 \times 3 \mathrm{~cm}$.

Small fragment of wall; fine thickened rounded outer rim; fine groove at junction between rim and wall; barbotine decoration: „drops”. Hard fine fabric with white inclusions, reddish yellow, Munsell 7.5YR 7/6; fine slip, on the outside upper body only, reddish yellow, Munsell 7.5YR 7/8.

95. MT; P50, K-5; fs. 4 × $2.6 \mathrm{~cm}$.

Small fragment of wall; barbotine decoration: "drops". Porous fabric with white inclusions, reddish yellow, Munsell 7.5YR 7/6; shiny reddish yellow slip, poorly preserved, outside, Munsell 7.5YR 6/8.

96. MT; P50, L, c.5; db. $8 \mathrm{~cm}$.

Fragment of wall; outside thickened beveled rim; fine groove at junction between rim and wall; barbotine decoration: "drops". Porous fabric with white inclusions, reddish yellow, Munsell 7.5YR 7/6; thick dull reddish yellow slip, Munsell 5YR6/6.

97. MT; P51, L $-9,-1.80-2,00 \mathrm{~m} ; \mathrm{db} .7 .5 \mathrm{~cm}$.

Fragment of wall; outside thickened beveled rim; fine groove at junction between rim and wall; barbotine decoration: "drops". Porous fabric with voids and white inclusions, reddish yellow, Munsell 5YR 7/6; thick dull reddish yellow slip, Munsell 5YR6/6.

98. MT; P 50, L, c. 5; fs. $4.2 \times 3 \mathrm{~cm}$.

Fragment of wall; outside thickened beveled rim; fine groove at junction between rim and wall; barbotine decoration: "drops". Porous fabric with voids and white inclusions, reddish yellow, Munsell 5YR 7/6; thick dull reddish yellow slip, Munsell 5YR6/6.

99. MT; P 50, j3; db. $8 \mathrm{~cm}$..

Fragment of wall; outside thickened beveled rim; fine groove at junction between rim and wall; barbotine decoration: "drops". Porous fabric with voids and white inclusions, reddish yellow, Munsell 5YR 7/6; thick dull reddish yellow slip, Munsell 5YR6/6.

100. MT; P 50, L 2; db. $8 \mathrm{~cm}$.

Fragment of wall; outside thickened beveled rim; fine groove at junction between rim and wall; barbotine decoration: "drops". Porous fabric with voids and white inclusions, reddish yellow, Munsell 5YR 7/6; thick dull reddish yellow slip, Munsell 5YR6/6.

101. MT; P. 50, j 12 ; fs. $3.6 \times 2.5 \mathrm{~cm}$.

Fragment of wall; barbotine decoration: "drops". Porous fabric with voids and white inclusions, reddish yellow, Munsell 5YR 7/6; thick dull reddish yellow slip, Munsell 5YR6/6.

102. MT; fs. 2 x $3.2 \mathrm{~cm}$.

Fragment. Outer beveled rim; fine groove at junction between rim and wall; barbotine decoration: „drops”. Porous hard sandy fabric with mica, reddish yellow, Munsell 5YR 7/6; fine shiny slip with pearly film, reddish yellow, Munsell 5YR7/8.

103. MT; P 49, H", VII, 18; fs. $2.6 \times 2.6 \mathrm{~cm}$.

Fragment of wall; outside thickened beveled rim; fine groove at junction between rim and wall; barbotine decoration: "drops". Porous fabric with voids and white inclusions, reddish yellow, Munsell 5YR 7/6; thick dull reddish yellow slip, Munsell 5YR6/6.

104. MT; P.; fs. 3.6 x $3.4 \mathrm{~cm}$. 
Fragment. Rounded wall, outside thickened beveled rim; groove at junction between rim and wall; barbotine decoration, „drops”. Porous fabric with voids and white inclusions, reddish yellow, Munsell 5YR 7/6; thick dull reddish yellow slip, Munsell 5YR6/6.

105. MT; P. 50, L 2, 5080; db. $7 \mathrm{~cm}$, df. $3.6 \mathrm{~cm}, \mathrm{~h} 7 \mathrm{~cm}$.

Restored (three quarter preserved, entire profile). Globular body; fine thickened rounded outer rim; rounded handle; fine groove at the junction between rim and wall; concave floor, inside; beveled ring with flat resting surface; barbotine decoration, „drops”, on the superior part of body. Hard fabric with fine send and white inclusions, poorly preserved, reddish yellow Munsell 7.5YR7/6; fine shiny slip, on the upper body, trickle on the floor, reddish yellow Munsell 7.5YR 7/8.

106. MT; P 50, L c3, db.8cm.

Three fragment of wall. Rounded wall, outside thickened beveled rim; groove at the junction between rim and wall; barbotine decoration, ,drops”. Porous fabric with voids and white inclusions, reddish yellow, Munsell 5YR 7/6; thick dull slip, reddish yellow, Munsell 5YR6/6.

107. MT, inv. no. 1535 ; P. 50 , k VII; fs. 6 x $3.5 \mathrm{~cm}$.

Fragment. Rounded wall, outside thickened beveled rim; groove at junction between rim and wall; rounded handle; barbotine decoration, ,drops”. Porous fabric with voids and white inclusions, reddish yellow, Munsell 5YR 7/6; thick dull slip, reddish yellow, Munsell 5YR6/6.

108. MT; P. 50, j 5. db. $8 \mathrm{~cm}$.

Fragment. Rounded wall, outside thickened beveled rim; groove at junction between rim and wall; barbotine decoration, "drops". Porous fabric with voids and white inclusions, reddish yellow, Munsell 5YR 7/6; thick shiny slip, reddish yellow, Munsell 5YR6/6.

109. MT; P. 50. L 4, 282; db. $7.5 \mathrm{~cm}$.

Fragment. Rounded wall, outside thickened beveled rim; groove at junction between rim and wall; barbotine decoration, "drops". Porous fabric with voids and white inclusions, reddish yellow, Munsell 5YR 7/6; thick dull slip on the upper body only, reddish yellow, Munsell 5YR6/6.

Ref: Teodor 1994, cat. 37, p. 88, fig. 4/6; Vulpe, Teodor 2003, cat. 830, p. 326, fig. $238 / 6$.

110. MT; P. 50, L, c2; db. $8 \mathrm{~cm}$.

Fragment. Rounded wall, outside thickened beveled rim; groove at junction between rim and wall; barbotine decoration, "drops". Porous fabric with voids and white inclusions, yellow, 10YR 8/6; thick shiny slip on the upper body only, with pearly film, yellow, Munsell 10YR 8/8.

111. MT, inv. no. 1229; P.; db.7.5cm.

Rounded wall, outside thickened beveled rim; groove at junction between rim and wall; barbotine decoration, "drops". Porous fabric with voids and white inclusions, reddish yellow, Munsell 5YR 7/6; thick shiny slip, reddish yellow, Munsell 5YR6/6.

112. MT, inv. no. 1230; P.; db. $7.5 \mathrm{~cm}$.

Fragment. Rounded wall, outside thickened beveled rim; groove at junction between rim and wall; barbotine decoration, "drops". Porous fabric with voids and white inclusions, reddish yellow, Munsell 5YR 7/6; thick shiny slip, reddish yellow, Munsell 5YR6/6.

113. MT, inv. no. 2280; $\mathrm{P} 87, \mathrm{~N} 1,-1.40 \mathrm{~m} ; \mathrm{db} .8 \mathrm{~cm}$.

Fragment. Rounded wall, outside thickened beveled outturned rim; two groove at the junction between rim and wall; barbotine decoration, "drops". Porous fabric with sand and mica, 
reddish yellow, Munsell 5YR 7/6; thick shiny slip with pearly film, reddish yellow, Munsell 5YR7/8.

Ref: Teodor 1994, p. 87, cat. 33, fig. 4/2; Teodor, Vulpe 2003, p. 326, cat. 826, fig. $238 / 2$.

114. MT; P. 49, H", VII; db. 7.5cm; pl. 4/114.

Fragment. Rounded wall, outside thickened beveled rim; groove at junction between rim and wall; barbotine decoration, "drops". Porous fabric with voids and white inclusions, reddish yellow, Munsell 5YR 7/6; thick dull slip, reddish yellow, Munsell 5YR6/6.

115. MT, inv. no. 3524; P.; db. $8 \mathrm{~cm}$.

Fragment. Rounded wall, outside thickened beveled rim; groove at junction between rim and wall; barbotine decoration, "drops". Homogeneous thick dull fabric, reddish yellow, Munsell 5YR 7/6; thick dull slip, on the outside floor missing, reddish yellow, Munsell 5YR7/6.

116. MT; P. 50, $\mathrm{J}^{2},-2 ; \mathrm{db} .8 \mathrm{~cm}$.

Fragment. Rounded wall, outside thickened beveled rim; groove at junction between rim and wall; handle trace; barbotine decoration, "drops". Porous hard fabric with voids and white inclusions, reddish yellow, Munsell 5YR 7/6; thick shiny slip, reddish yellow, Munsell 5YR6/6.

117. MT; P 50, j c4; db. $8 \mathrm{~cm}$, hc. $6 \mathrm{~cm}$.

Fragment. Rounded wall, outside thickened beveled rim; groove at junction between rim and wall; barbotine decoration, "drops". Porous hard fabric with voids and white inclusions, reddish yellow, Munsell 7.5YR 7/6; thick shiny slip, reddish yellow, Munsell 7.5YR7/8.

118. MT; P 50, J 6; db. $8 \mathrm{~cm}$, hc. $5 \mathrm{~cm}$.

Fragment. Rounded wall, outside thickened beveled rim; groove at junction between rim and wall; rounded handle; barbotine decoration, "drops". Porous hard fabric with voids and white inclusions, reddish yellow, Munsell 7.5YR 7/6; thick shiny slip, reddish yellow, Munsell 7.5YR7/8.

119. MT; P 1257 , fs. 5 x $5 \mathrm{~cm}$.

Fragment. Rounded wall, outside thickened beveled rim; groove at junction between rim and wall; barbotine decoration, "drops". Porous hard fabric with voids and white inclusions, reddish yellow, Munsell 7.5YR 7/6; thick shiny slip, reddish yellow, Munsell 7.5YR7/8.

120. MT, inv. no. 2279 ; db. $7.5 \mathrm{~cm}$; hc. $4.9 \mathrm{~cm}$.

Fragment. Rounded wall, outside thickened beveled rim; groove at junction between rim and wall; barbotine decoration, "drops". Porous hard fabric with voids and white inclusions, reddish yellow, Munsell 5YR 7/6; thick shiny slip, reddish yellow, Munsell 5YR6/6.

121. MT, inv. no. 2283; P $85, \mathrm{~N}$; db. $7.5 \mathrm{~cm}$, hc. $2.9 \mathrm{~cm}$.

Fragment. Rounded wall, outside thickened beveled rim; groove at junction between rim and wall; barbotine decoration, "drops". Porous hard fabric with voids and white inclusions, reddish yellow, Munsell 7.5YR 7/6; thick shiny slip, reddish yellow, Munsell 7.5YR6/8.

122. MT; P.; 4.5 x $4.8 \mathrm{~cm}$.

Fragment. Rounded wall, outside thickened beveled rim; groove at junction between rim and wall; barbotine decoration, "drops". Porous hard fabric with voids and white inclusions, reddish yellow, Munsell 7.5YR 7/6; thick shiny slip, reddish yellow, Munsell 7.5YR6/8.

123. MT; P. 50, J5, db. $8 \mathrm{~cm}$; hc. $4 \mathrm{~cm}$.

Fragment. Rounded wall, outside thickened beveled rim; groove at junction between rim and wall; barbotine decoration, "drops". Porous hard fabric with voids and white inclusions, reddish yellow, Munsell 7.5YR 7/6; thick shiny slip, reddish yellow, Munsell 7.5YR7/8.

124. MT; P 50 L, c5, fs. 5 x $2.4 \mathrm{~cm}$. 
Fragment. Rounded wall, outside thickened beveled rim; groove at junction between rim and wall; barbotine decoration, "drops". Porous hard fabric with voids and white inclusions, yellow, Munsell 10YR 8/6; thick shiny slip, yellow, Munsell 10YR 8/8.

125. MT inv. no. 1242 ; P.; fs. $3.3 \times 4.2 \mathrm{~cm}$.

Fragment. Rounded wall, outside thickened beveled rim; groove at junction between rim and wall; barbotine decoration, "drops". Porous hard fabric with voids and white inclusions, yellow, Munsell 10YR 8/6; thick shiny slip, yellow, Munsell 10YR 8/8.

126. MT; P. 87, N 1, 1, m 49, 0.75m; db. $7.8 \mathrm{~cm}$, hc. $3.9 \mathrm{~cm}$.

Fragment. Rounded wall, outside thickened beveled rim; groove at junction between rim and wall; barbotine decoration, "drops". Porous hard fabric with voids and white inclusions, reddish yellow, Munsell 7.5YR 7/6; thick shiny slip, strong brown, Munsell 7.5YR 5/8.

127. MT; P. 50, L c3; $3.3 \times 2 \mathrm{~cm}$.

Fragment. Rounded wall, outside thickened beveled rim; groove at junction between rim and wall; barbotine decoration, "drops". Porous fabric with voids and white inclusions, yellow, Munsell 10YR 8/6; thick shiny slip, yellow, Munsell 10YR 8/8.

128. MT; inv. no. 1535; P. 50 k, VII; dim. fr. 5 x $4.2 \mathrm{~cm}$.

Fragment. Rounded wall; groove at junction between rim and wall; barbotine decoration, "drops". Porous fabric with voids and white inclusions, reddish yellow, Munsell 7.5YR 7/6; thick shiny slip, reddish yellow, Munsell 7.5YR 7/8.

129. MT; P 50, $\mathrm{J}^{2},-2$.

Fragment. Rounded wall; groove at junction between rim and wall; fragmentary rounded handle; barbotine decoration, "drops". Porous fabric with voids and white inclusions, reddish yellow, Munsell 5YR 7/6; thick dull slip, reddish yellow, Munsell 5YR6/6.

130. MT; P. 50, J 7; fs. 5 x 4,5cm.

Fragment. Rounded wall; groove at junction between rim and wall; barbotine decoration, "drops". Porous fabric with voids and white inclusions, reddish yellow, Munsell 7.5YR 7/6; thick shiny slip, reddish yellow, Munsell 7.5YR 7/8.

131. MT; P. 50, Lc3, i 8/44; db. 8 m.

Fragment. Rounded wall; groove at the junction between rim and wall; barbotine decoration, "drops". Porous fabric with voids and white inclusions, yellow, Munsell 10YR8/6; thick shiny slip, poorly preserved, yellow, Munsell 10YR 7/8.

132. MT; P $50 \mathrm{~K}$, VII, 1535 , fs. 3.8 x $4.6 \mathrm{~cm}$.

Fragment. Rounded wall; groove at junction between rim and wall, outside; barbotine decoration, "drops". Porous fabric with voids and white inclusions, yellow, Munsell 10YR 8/6; thick shiny slip, reddish yellow, Munsell 10YR 8/8.

133. MT; P., fs. $2.5 \times 2.8 \mathrm{~cm}$.

Fragment. Rounded wall; groove at the junction between rim and wall, outside; barbotine decoration, "drops". Porous fabric with voids and white inclusions, yellow, Munsell 10YR 8/6; thick shiny slip, reddish yellow, Munsell 10YR 8/8.

134. MT; P 90, P', १ 35, $-1.30 \mathrm{~m} ;$ db. $8 \mathrm{~cm}$.

Fragment. Rounded wall; groove at junction between rim and wall, outside; barbotine decoration, "drops". Porous fabric with voids and white inclusions, yellow, Munsell 10YR 8/6; thick shiny slip, reddish yellow, Munsell 10YR 8/8.

135. MT; P. 50, K 3, db. $8 \mathrm{~cm}$.

Fragment. Rounded wall; groove at the junction between rim and wall, outside; barbotine decoration, "drops". Porous fabric with voids and white inclusions, yellow, Munsell 10YR 8/6; thick shiny slip, reddish yellow, Munsell 10YR 8/8. 
136. MT; P. 50, J8; fs. $3.6 \times 3.5 \mathrm{~cm}$.

Fragment. Rounded wall; groove at junction between rim and wall, outside; barbotine decoration, "drops". Porous fabric with voids and white inclusions, yellow, Munsell 10YR 8/6; thick shiny slip, reddish yellow, with brown patches, Munsell 10YR 8/8.

137. MT; P. 2283, fs. $3 \times 3.5 \mathrm{~cm}$.

Fragment. Rounded wall; groove at junction between rim and wall, outside; barbotine decoration, "drops". Porous fabric with voids and white inclusions, yellow, Munsell 10YR 8/6; thick shiny slip, reddish yellow, with brown patches, Munsell 10YR 8/8.

138. MT; P. fs. $3.2 \times 3.6 \mathrm{~cm}$.

Fragment. Rounded wall; groove at junction between rim and wall, outside; barbotine decoration, "drops". Porous fabric with voids and white inclusions, yellow, Munsell 10YR 8/6; thick shiny slip, reddish yellow, Munsell 10YR 8/8.

139. MT; P. $50, \mathrm{~J}^{2},-2$; fs. $2.6 \times 5.2 \mathrm{~cm}$.

Fragment. Rounded wall; groove at junction between rim and wall, outside; barbotine decoration, "drops". Porous fabric with voids and white inclusions, yellow, Munsell 10YR 8/6; thick shiny slip, reddish yellow, Munsell 10YR 8/8.

140. MT; fs. $2.5 \times 3.6 \mathrm{~cm}$.

Fragment. Rounded wall; groove at junction between rim and wall, outside; barbotine decoration, "drops". Porous fabric with voids and white inclusions, yellow, Munsell 10YR 8/6; thick shiny slip, reddish yellow, Munsell 10YR 8/8.

141. MT; P. 50, KE, c5, fs. 4 x $3.2 \mathrm{~cm}$.

Fragment. Rounded wall; groove at junction between rim and wall, outside; barbotine decoration, "drops". Porous fabric with voids and white inclusions, reddish yellow, Munsell 7.5YR 7/6; thick shiny slip, reddish yellow, Munsell 7.5YR 6/8.

142. MT; P50, J5; fs. 6 x 5cm.

Fragment. Rounded wall; inside concave floor; beveled ring with resting surface flat; barbotine decoration, "drops". Porous fabric with voids and white inclusions, reddish yellow, Munsell 7.5YR 7/6; thick shiny slip, reddish yellow, Munsell 7.5YR 7/8, on the outside upper body, only.

143. MT; P 1951, L 0,60m; db. 8 cm; hc. $5.3 \mathrm{~cm}$.

Fragment. Globular body; outside thickened beveled rim, slightly outturned; groove at junction between rim and wall, outside; rounded handle; barbotine decoration, "wheat grains". Compact hard fabric with white inclusions, yellow, Munsell 10YR8/6; fine shiny slip, poorly preserved, yellow, Munsell 10YR 7/8.

144. MT; P. 51, L', 0.60m; fs. 4 x $2.5 \mathrm{~cm}$.

Fragment. Globular body; outside thickened beveled rim, slightly outturned; groove at junction between rim and wall, outside; barbotine decoration, "wheat grains".

Compact hard fabric with white inclusions, yellow, Munsell 10YR8/6; fine shiny slip, poorly preserved, yellow, Munsell 10YR 7/8.

145. MT; db. $8 \mathrm{~cm}$.

Fragment. Globular body; outside thickened beveled rim, slightly outturned; groove at junction between rim and wall, outside; barbotine decoration, "wheat grains".

Compact hard fabric with white inclusions, yellow, Munsell 10YR8/6; fine shiny slip, poorly preserved, yellow, Munsell 10YR 7/8.

146. MT; P. 1236; fs. 4.8 x $3.8 \mathrm{~cm}$. pl. $4 / 146$.

Fragment. Globular body; outside thickened beveled rim, slightly outturned; groove at junction between rim and wall, outside; barbotine decoration, "wheat grains". 
Compact hard fabric with white inclusions, yellow, Munsell 10YR8/6; fine shiny slip, poorly preserved, yellow, Munsell 10YR 7/8.

147. MT, inv. no. 1231; P.; db. $8 \mathrm{~cm}$.

Fragment. Globular body; outside thickened beveled rim, slightly outturned; groove at junction between rim and wall, outside; barbotine decoration, "wheat grains".

Porous fabric with sand and mica, reddish yellow, Munsell 5YR 7/6; fine shiny slip, with pearly film, reddish yellow, Munsell 5YR7/8; fingerprint, outside.

148. MT, db. $10 \mathrm{~cm}$.

Fragment. Globular body; outside thickened beveled rim, slightly outturned; groove at junction between rim and wall, outside; barbotine decoration, "wheat grains".

Compact hard fabric with white inclusions, yellow, Munsell 10YR8/6; fine shiny slip, poorly preserved, yellow, Munsell 10YR 7/8.

149. MT; P. 50, HJ; fig. 4/149.

Fragment. Globular body; outside thickened beveled rim, slightly outturned; groove at junction between rim and wall, outside; barbotine decoration, "leaves and drops"

Porous fabric with sand and mica, reddish yellow, Munsell 5YR 7/6; fine shiny slip, with pearly film, reddish yellow, Munsell 5YR7/8.

150. MT; db. $8 \mathrm{~cm}$, df $3.7 \mathrm{~cm}$, h $8 \mathrm{~cm}$; pl. $4 / 150$.

Restored (half preserved; profile entire). Globular body; outside thickened beveled rim, slightly outturned; groove at the junction between rim and wall, outside; slightly concave floor inside; rounded handle. Compact fabric with sand and white inclusions, reddish yellow, Munsell 7.5YR7/8; fine shiny slip, uneven applied, reddish yellow, Munsell 5YR7/8.

151. MT; P. 90, $\mathrm{P}^{\prime},-1.50 \mathrm{~m}$; db. $7.5 \mathrm{~cm}$.

Fragment. Globular body; outside thickened beveled rim, slightly outturned; groove at junction between rim and wall, outside. Porous fabric with voids and white inclusions, reddish yellow, Munsell 5YR 7/6; thick shiny slip, reddish yellow Munsell 5YR6/6.

152. MT; P. $49 ; 3.5 \times 3.5 \mathrm{~cm}$.

Fragment. Globular body; outside thickened beveled rim; groove at the junction between rim and wall, outside; handle trace. Porous fabric with voids and white inclusions, reddish yellow, Munsell 7.5YR 7/6; thick shiny slip, reddish yellow, Munsell 7.5YR 6/8.

153. MT; P. L I-J; db. $8 \mathrm{~cm}$.

Fragment. Globular body; outside thickened beveled rim; groove at junction between rim and wall, outside. Porous fabric with voids and white inclusions, yellow, Munsell 10YR 8/6; fine shiny slip, yellow with brown patches, Munsell 10YR 8/8.

154. MT; P 50, L 2; fs. 4 x $3 \mathrm{~cm}$.

Fragment. Globular body; outside thickened beveled rim; groove at junction between rim and wall, outside. Compact fine fabric, reddish yellow, Munsell 7.5YR 7/6; fine shiny slip, reddish yellow, Munsell 7.5YR 7/8.

155. MT; P 50, L5; db. $8 \mathrm{~cm}$.

Fragment. Globular body; outside thickened beveled rim; groove at junction between rim and wall, outside. Porous fabric with voids and white inclusions, yellow, Munsell 10YR 8/6; fine shiny slip, yellow, Munsell 10YR 7/8.

156. MT; P 50 I III - IV; db. 8cm.

Fragment. Globular body; outside thickened beveled rim; groove at junction between rim and wall, outside. Compact fine fabric, yellow, Munsell 10YR 8/8; fine shiny slip, Munsell 10YR 7/8.

157. MT inv. no. 1239 (3529); $\mathrm{P} . ; \mathrm{db} 8 \mathrm{~cm}$. 
Fragment. Globular body; outside thickened beveled rim; groove at junction between rim and wall, outside; handle trace. Hard fabric with voids and white inclusions, reddish yellow, Munsell 5YR 7/6; thick shiny slip on the outside upper body, trickle on the floor, reddish yellow, Munsell 5YR6/6.

158. MT; P 50, KVII, db. $9 \mathrm{~cm}$.

Fragment. Globular body; outside thickened beveled rim; groove at junction between rim and wall, outside. Porous hard fabric with voids and white inclusions, reddish yellow, Munsell 5YR 7/6; thick shiny slip, reddish yellow, Munsell 5YR6/6.

159. MT; P 90, $P^{\prime} \uparrow \quad 36,-1.30 \mathrm{~m}, \mathrm{db} .9 \mathrm{~cm}$.

Fragment. Globular body; outside thickened beveled rim; groove at junction between rim and wall, outside; rounded handle. Porous fabric with voids and white inclusions, reddish yellow, Munsell 5YR 7/6; slip entire missing.

160. MT; P 50, j c4; db. $8 \mathrm{~cm}$, h. $3.5 \mathrm{~cm}$.

Fragment, globular slightly beveled body; outside thickened beveled rim; groove at junction between rim and wall, outside; rounded handle. Porous hard fabric with voids and white inclusions, reddish yellow, Munsell 5YR 7/6; thick shiny slip, reddish yellow, Munsell 5YR6/6.

161. MT; P 85, Ni 16, 1.60; db. 9cm; pl. 4/161.

Fragment, globular slightly beveled body; outside thickened beveled rim; groove at junction between rim and wall, outside; rounded handle. Porous hard fabric with voids and white inclusions, reddish yellow, Munsell 5YR 7/6; thick shiny slip, reddish yellow, Munsell 5YR6/6.

162. MT; fs. $3.2 \mathrm{~cm}$.

Fragment. Globular body; slightly concave inside floor with nipple, outside; fine beveled low ring with flat resting surface. Compact hard fabric, reddish yellow, Munsell 7.5YR 7/6; shiny slip, poorly preserved, on the upper body, strong brown, Munsell 7.5YR 5/8.

163. MT, inv. no. 2288; P 90; P', c. 1-2, L4; df. 3.4cm.

Fragment. Slightly concave inside floor with nipple, outside; fine beveled low ring with flat resting surface. Hard fabric with voids and white inclusions, reddish yellow, Munsell 7.5 YR7/6.

164. MT; P. 1226, df. $3.5 \mathrm{~cm}$.

Fragment. Slightly concave inside floor with nipple, inside; fine beveled low ring with flat resting surface. Compact hard fabric, reddish yellow, Munsell 7.5YR 7/8.

165. MT; P 1927-1928, df. $4.7 \mathrm{~cm}$.

Fragment. Slightly concave inside floor with nipple, inside; fine beveled low ring with flat resting surface. Porous fabric with white inclusions, reddish yellow, Munsell 7.5YR 7/8; shiny slip, inside, reddish yellow, Munsell 7.5YR 6/8.

166. MT, inv. no. 1249; P.; df. $4.2 \mathrm{~cm}$.

Fragment. Slightly concave inside floor with prominent nipple, inside; fine beveled low ring with flat resting surface. Compact hard fabric, reddish yellow, Munsell 7.5YR 7/6; reddish yellow slip, poorly preserved, Munsell 7.5YR 7/8.

167. MT; P. 50, L, c4, df. $3.5 \mathrm{~cm}$.

Fragment. Slightly concave inside floor with prominent nipple, inside and flat nipple, outside; fine beveled low ring with flat resting surface. Hard reddish yellow fabric, Munsell 7.5 YR $7 / 6$.

168. MT; Poiana 1928-1928, df. $4 \mathrm{~cm}$. 
Fragment. Slightly concave inside floor with prominent nipple, outside; fine beveled low ring with flat resting surface. Porous hard fabric with voids and white inclusions, reddish yellow, Munsell 5YR 7/6.

169. MT; P. 50 , jc; df. $3.5 \mathrm{~cm}$.

Fragment. Globular body; slightly concave inside floor fine beveled low ring; handle trace. Porous hard fabric with voids and white inclusions, reddish yellow, Munsell 5YR 7/6; fine shiny reddish yellow slip on the outside upper body, only, Munsell 5YR6/6.

170. MT; P 51, L', c4; df. $3.5 \mathrm{~cm}$.

Fragment. Slightly concave inside floor with prominent nipple outside, and flat nipple, inside; fine beveled low ring with flat resting surface. Porous hard fabric with voids and white inclusions, reddish yellow, Munsell 5YR 7/6; fine shiny slip, poorly preserved, reddish yellow, Munsell 5YR6/6.

171. MT; P 50, L c3, $\uparrow$ 3/43. df. $4 \mathrm{~cm}$.

Fragment. Globular body; slightly concave inside floor; fine beveled low ring; handle trace; fine outside groove at junction between floor and ring. Hard vitreous fabric, similar with 6-7, 18 and 32, reddish yellow, Munsell 7.5 YR 7/6; fine shiny slip, cover the whole inside and upper body, outside; reddish yellow, inside Munsell 7.5 YR 6/8; very dark brown, outside Munsell 7.5 YR 2.5/2.

172. MT; P. 50 k, 0.40 ; df. $4 \mathrm{~cm}$.

Fragment. Globular body; slightly concave inside floor; fine beveled low ring; fine outside groove at the junction between floor and ring. Porous fabric with voids and white inclusions, reddish yellow, Munsell 5YR 7/6; fine shiny slip, poorly preserved, reddish yellow, Munsell 5YR6/6.

173. MT; P. 49, H, 1.00m; df. $4 \mathrm{~cm}$.

Fragment. Globular body; slightly concave inside floor; fine beveled low ring. Porous fabric with voids and white inclusions, reddish yellow, Munsell 5YR 7/6; fine shiny slip, poorly preserved, reddish yellow, Munsell 5YR6/6.

174. MT, inv. no. 6157 ; P.; df. $3.5 \mathrm{~cm}$.

Fragment. Globular body; slightly concave inside floor with prominent nipple outside; fine beveled low ring; fine outside groove at junction between floor and ring. Porous fabric with voids and white inclusions, reddish yellow, Munsell 5YR 7/6; fine shiny slip, poorly preserved, reddish yellow, Munsell 5YR6/6.

175. MT; P. 50 J; df. $3.5 \mathrm{~cm}$.

Fragment. Globular body; slightly concave inside floor with prominent nipple, outside; fine beveled low ring. Porous fabric with voids and white inclusions, reddish yellow Munsell 7.5YR 7/6; fine shiny slip, on the upper body, poorly preserved, reddish yellow, Munsell 7.5YR 7/8.

176. MT; P. $49 \mathrm{H} \mathrm{V},-1.50 \mathrm{~m}$; df. $3.5 \mathrm{~cm}$.

Fragment. Globular body; slightly concave inside floor with prominent nipple outside; fine beveled low ring. Porous fabric with mica, reddish yellow Munsell 7.5YR 7/6; fine shiny slip, on the upper body, poorly preserved, reddish yellow, Munsell 7.5YR 7/8.

177. MT; P., df. $3.5 \mathrm{~cm}$.

Fragment. Globular body; slightly concave inside floor; Porous fabric with mica, reddish yellow Munsell 7.5YR 7/6; fine shiny slip, on the upper body, poorly preserved, reddish yellow, Munsell 7.5YR 7/8.

178. $M T$, nr. inv. 2277; P 90, $\mathrm{P}^{\prime}, 91-2$. df. $3.5 \mathrm{~cm}$. 
Fragment. Globular body; slightly concave inside floor with prominent nipple inside, flat nipple, outside; fine beveled low ring. Compact hard fabric, reddish yellow, Munsell 7.5YR 7/6; reddish yellow slip, poorly preserved Munsell 7.5YR.

179. MT; P.; db. $8 \mathrm{~cm}$.

Fragment. Globular body; outside thickened beveled outer rim; groove at the junction between rim and wall, outside; rounded handle. Homogeneous reddish yellow fabric, Munsell 7.5YR 7/6; fine dull reddish yellow slip Munsell 7.5 YR7/8.

180. MT; P. 50, J8; fs. $4.2 \times 3 \mathrm{~cm}$.

Fragment. Globular body; rounded handle. Porous fabric, with voids and white inclusions, yellow, Munsell 10YR 8/6; shiny yellow slip, Munsell 10YR 8/8.

181. MT; P. 50 L 2; fs. 3.2 x $4.2 \mathrm{~cm}$;

Fragment. Globular body; beveled slightly outer rim; rounded handle. Homogeneous yellow fabric, Munsell 10YR 8/6; shiny yellow slip, Munsell 10YR 8/8.

182. MT; P. 50, J 3; fs. $3.5 \times 5.2 \mathrm{~cm}$.

Fragment. Globular body; rounded handle. Porous fabric, with voids and white inclusions, yellow, Munsell 10YR 8/6; shiny yellow slip, Munsell 10YR 8/8.

183. MT, inv. no. 1258 ; db. 12 cm.; pl. 4/183.

Fragment; with rounded rim, straight; "collar" concave on the inside, globular body; groove at the junction between "collar" and wall, outside; one fragmentary handle, slightly heightened. Fine reddish yellow fabric, Munsell 5YR7/8; shiny slip, reddish brown Munsell 5YR 5/4.

184. MT, inv. no. 2297 ; db. $12 \mathrm{~cm}$.

Fragment; with rounded rim, straight; "collar" concave on the inside, globular body; groove at the junction between "collar" and wall, outside. Fine reddish yellow fabric, Munsell 5YR7/8; shiny slip, reddish brown Munsell 5YR 5/4.

\section{BIBLIOGRAPHY}

Glodariu 1974 - I. Glodariu, Relațiile comerciale ale Daciei cu lumea elenistică şi romană, Cluj. Hayes 1973 - J. W. Hayes, The Roman Pottery from South Stoa at Corinth, Hesperia 42, 4, 416-470. Hayes 1976 - J. W. Hayes, Roman Pottery in the Royal Ontario Museum, Toronto.

Hayes 1985 - J. W. Hayes, Sigillate orientali, Enciclopedia dell'arte antica classica e orientale. Atlante delle forme ceramiche. II. Ceramica fine Romana nell basino Mediteraneo (tardo-ellenismo e primo impero), Roma, 2-95.

Hayes 1997 - J. W. Hayes, Handbook of Mediterranean Roman Pottery, British Museum Press/ University of Oklahoma Press, Great Britain.

Khrschanovskiy 1998 - V. Khrschanovskiy, The burials of the ${ }^{1 s t}-2^{\text {nd }}$ Centuries $A D$ with red slip pottery from Iluraton necripolis, Papers of the satate Historical Museum, vol.102, Hellenistic and Roman pottery in the Northern Pontic Area I, Moscow, 77-88.

Suceveanu 2000 - Al. Suceveanu, La céramique romaine des I-er - III -e siècles ap. J.-C, Histria X, Bucureşti.

Teodor 1994 - S. Teodor, Ceramica de import din aşezarea geto-dacică de la Poiana, jud. Galați, I, Carpica 25, 73- 123.

Vulpe, Vulpe 1927-1932 - R. Vulpe, E. Vulpe, La fouilles de Poiana, Dacia 3-4, 253-351.

Vulpe 1951 - R. Vulpe, Raport preliminar asupra activității şantierului arheologic Poiana - Tecuci, 1950, SCIV 2, 1, 178-180.

Vulpe 1957 - R. Vulpe, La civilisation dace et ses problémes á la lumière des dernières foilles de Poiana, en Basse - Moldavie, Dacia N.S. 1, 143-165. 
Vulpe, Teodor 2003 - R. Vulpe, S. Teodor, Piroboridava. Aşezarea geto-dacică de la Poiana, Bucureşti.

Zhuravlev 2002 - D.V. Zhuravlev., Terra Sigillata and red slip pottery in the north pontic region (A short bibliographical survey), Ancient civilization from Scythia to Siberia, vol. 8, nr. 3-4, sept. 2002, 237-309.

\section{ABBREVIATIONS}

Atlante II - Atlante delle forme ceramiche. II. Enciclopedia dell'arte antica. Classica e orientale. Roma, 1985.

Munsell - Munsell Color Charts for plant tissue, 1994

Muzeums

MT - City Museum, Tecuci.

MNA=MNIR - National Museum of Romanian History, Bucharest

\section{OTHER ABBREVIATIONS}

$\uparrow$ - square

db. - rim diameter

df. - ring diameter

h/i- height

hc - height preserved

L/ loc. - house

$\mathbf{S}-$ section

df.- dimensiuni fragment

inv. no. - inventory number

fs. - fragment size

Ref. - reference 


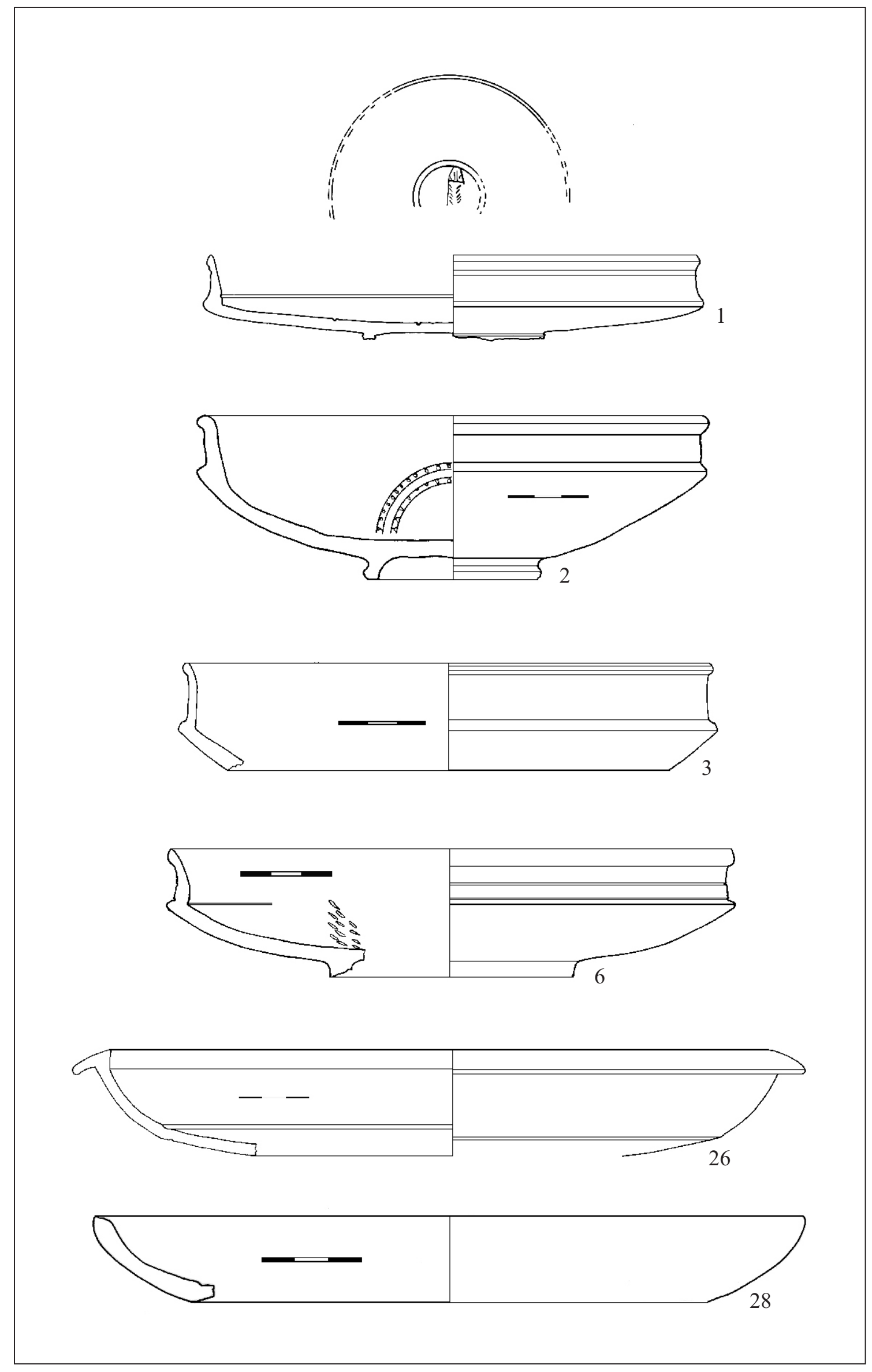

Pl. 1 

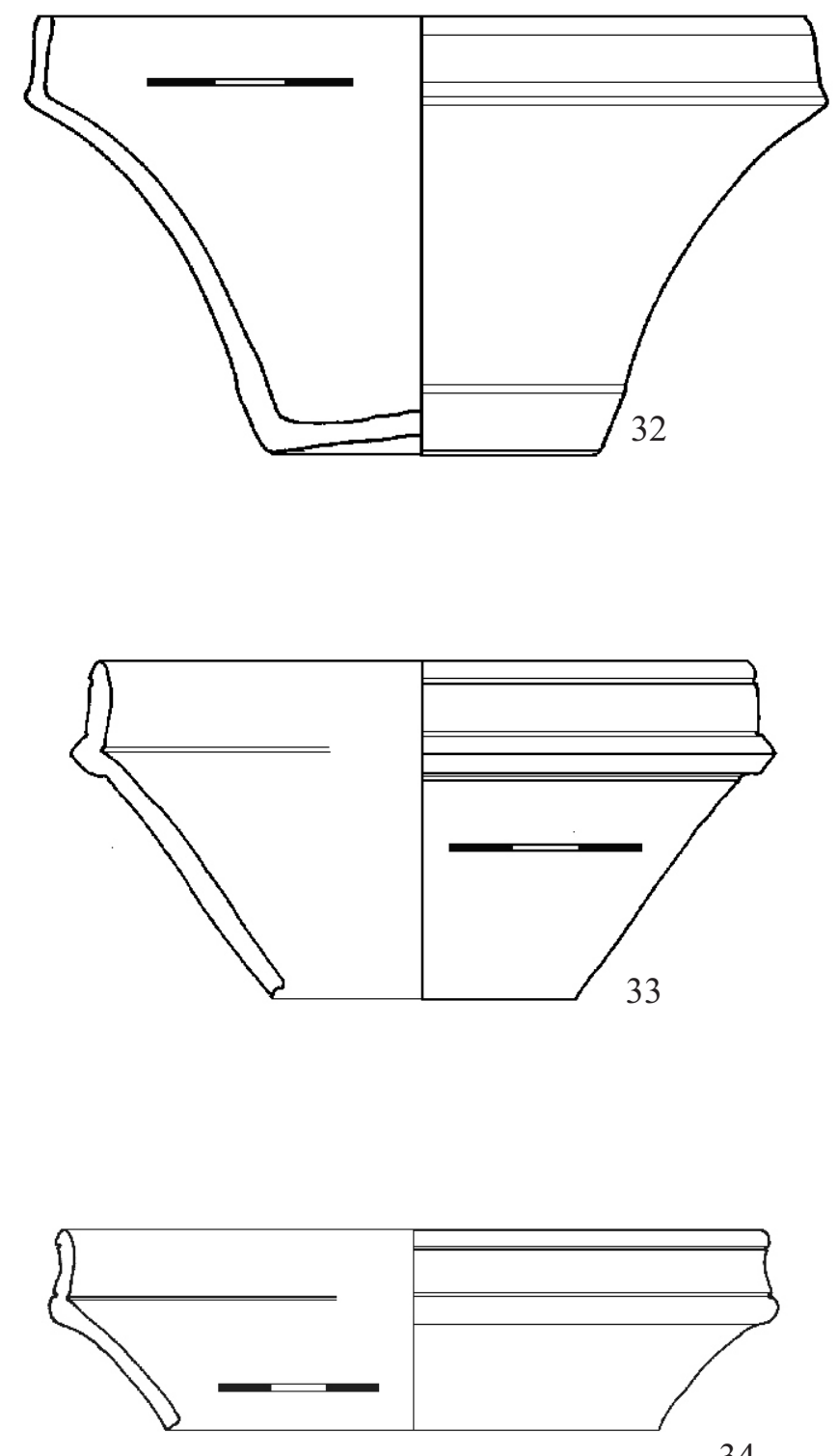

34

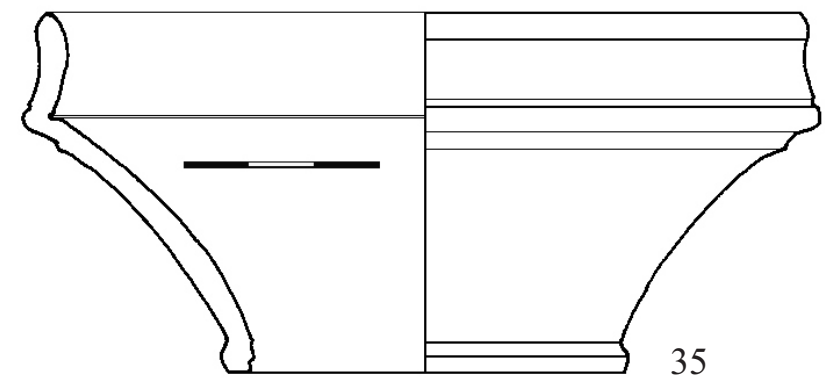

PI. 2 


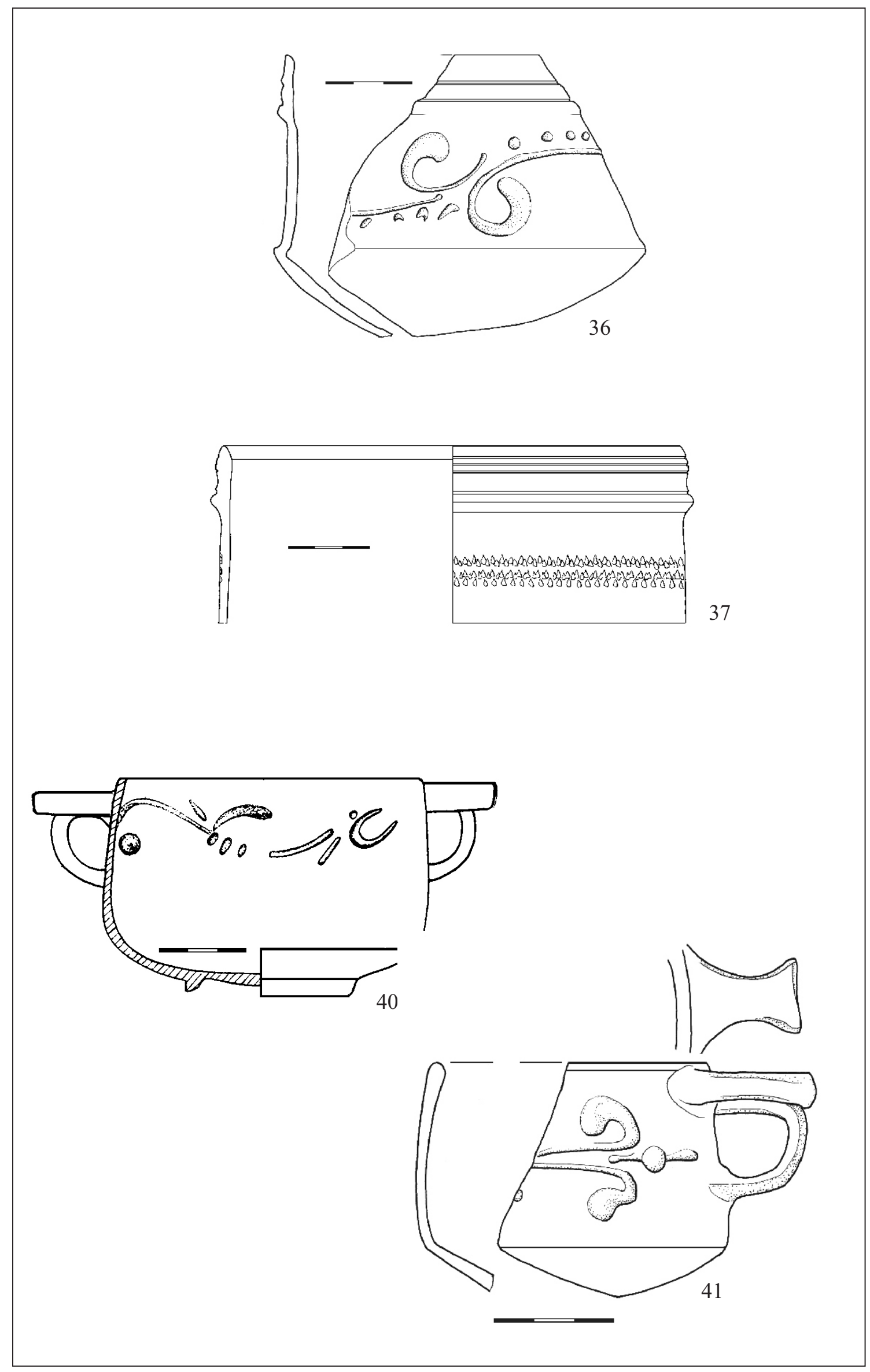

Pl. 3 


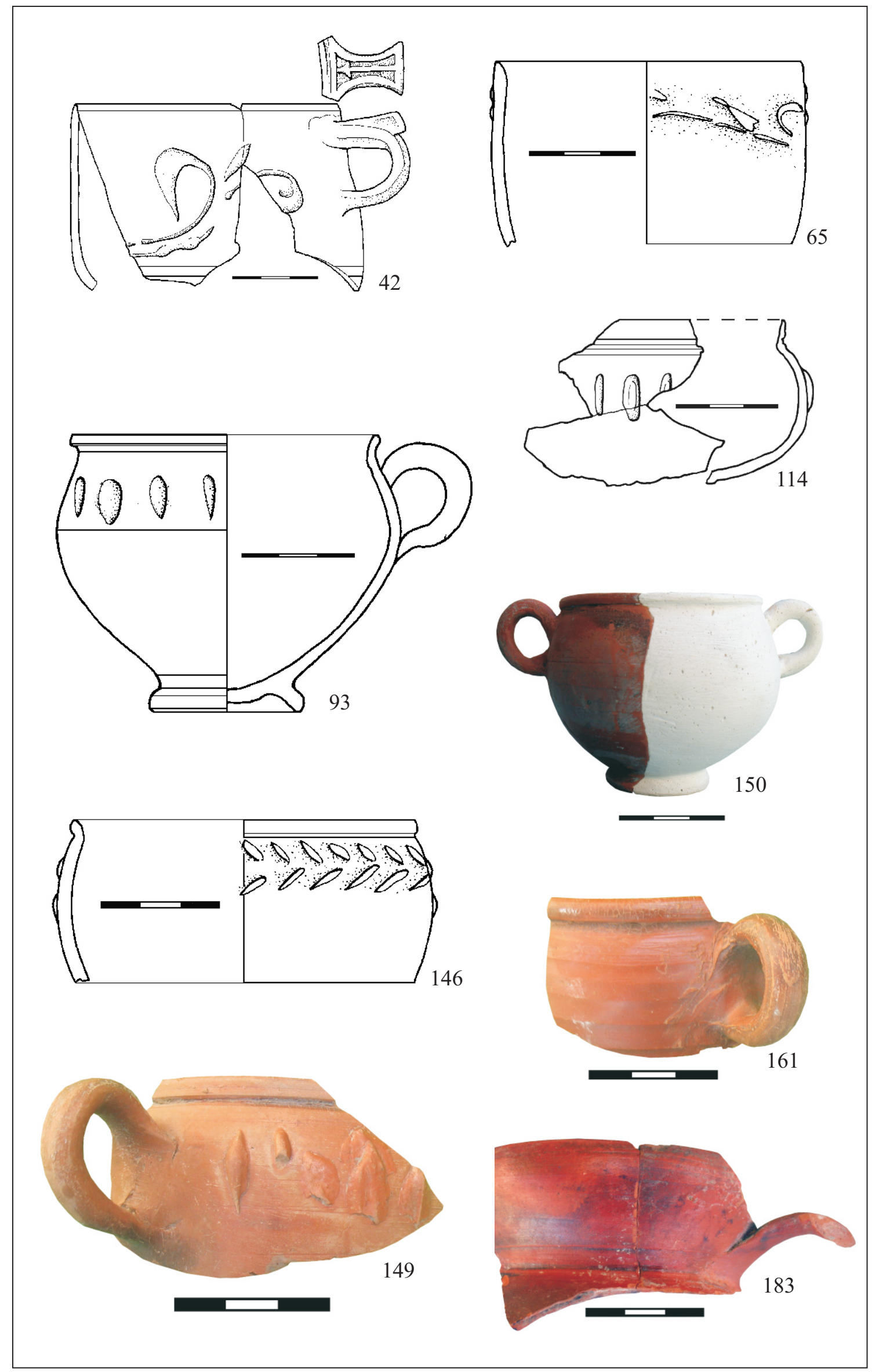

Pl. 4 